\title{
Spin-boson description of electron transmission through a molecular wire ${ }^{\text {is }}$
}

\author{
E.G. Petrov ${ }^{a, *}$, V. May ${ }^{b}$, P. Hänggi ${ }^{c}$ \\ ${ }^{a}$ Bogolyubov Institute for Theoretical Physics, National Academy of Sciences of Ukraine, Metrologichna str., 14-b, UA-03143 Kiev, Ukraine \\ ${ }^{\mathrm{b}}$ Institut für Physik, Humboldt Universität zu Berlin, Newtonstraße 15, D-12489 Berlin, Germany \\ ${ }^{\mathrm{c}}$ Institut für Physik, Universität Augsburg, D-86135 Augsburg, Germany
}

Received 27 June 2003; accepted 17 September 2003

\begin{abstract}
A spin-boson model based on local pseudo-spins acting in the electronic occupation number space is suggested and used to describe the thermally activated interelectrode current through a molecular wire. Utilizing the density matrix technique a unified description is achieved of all those kinetic processes which contribute to the inelastic current formation. These processes cover a specific superexchange transition between the terminal wire units as well as all sequential transitions leading from a given wire unit to the neighboring one. The exact solution of the kinetic equations is applied to a detailed analysis of the wire-length dependence of the current. The behavior differs between the sequential and the superexchange transfer mechanism and is discussed for a fixed electric field-strength in the wire as well as for a fixed applied voltage.
\end{abstract}

(C) 2003 Elsevier B.V. All rights reserved.

PACS: 05.20.Dd; 05.60.+w

Keywords: Interelectrode current; Kinetic equations; Molecular wire; Voltage bias

\section{Introduction}

Originally, the spin-boson model has been introduced as a generic description of particle or quasi-particle transfer in two-level systems coupled to a dissipative environment with the latter described as huge set of independent harmonic oscillators [1-4]. This model attracted particular interest when studying the driving by external regular and stochastic fields [5-8]. However, the outstanding importance of the spin-boson model is mainly related to the fact that a large variety of results do exist. A generalization of the spin-boson model to multi-level systems can be found at different places (cf., e.g. $[6,9,10])$. In the present paper, we will carry out such

\footnotetext{
Dedicated to the 60th birthday of Ulrich Weiss.

* Corresponding author. Tel.: +44-266-9141; fax: +44-266-5998.

E-mail addresses: epetrov@bitp.kiev.ua (E.G. Petrov), may@physik.hu-berlin.de (V. May), hanggi@physik.uni-augsburg.de (P. Hänggi).
}

a generalization to describe the inelastic charge transmission through molecular wires $[11,12]$.

Intensive research work has been invested on the study of molecular wires, i.e., single molecules or polymer strands embedded in between two electrodes (for a recent reviews, see [13-21]). These diversified activities are not only caused by the fundamental interest on electron transfer (ET) processes in single molecules or solid-state molecule hybrid structures. Further motivation has also been originated by the long-standing wish to control micro-currents in molecular devices via, e.g., rectification or gating [11,12,22-28]. Recent experimental results on the conductivity of single molecules [29-34] may serve as a good starting point to clarify the physical mechanisms of such a control.

Most of the theoretical approaches on molecular wires focused on the description of an elastic current in utilizing the Landauer-Büttiker theory $[35,36]$. In this approach charge motion trough the molecular wire is described as a multitude of elastic scattering processes $[20,28,30,31,37]$. If inelastic processes contribute to the 
overall current what will be the case whenever dephasing processes within wire units destroy the ET coherency the problem becomes more involved [14,17,38-44].

The investigation of the current-voltage $(I-V)$ characteristics of a molecular wire combined with a study of its wire-length dependency became an important way to reveal the mechanism of charge transmission. It is wellknown from studies on ET reactions in molecular complexes that fast nuclear relaxation processes result in the sequential (hopping) mechanism of ET along the molecular chain (see, e.g. [45]). In the case of a molecular wire and if a small voltage $V$ is applied this mechanism is responsible for the Ohmic regime of ET. Now, the dependence of the wire conductance on the number of wire units $N$ is proportional to the factor $\sim[1+\xi(N-1)]^{-1}$ [41]. A similar dependence (like $\sim[A+B(N-1)]^{-1}$ ) has been proposed by Nitzan and co-workers [46] to fit data on the $N$-dependency of an overall steady-state rate constant. Moreover, Bixon and Jortner [47] used such types of expressions to describe long-range charge transfer reactions in DNA stacks.

The other basic ET mechanism may become observable in those molecular wires where the energy gaps between the wire LUMO-levels and the Fermi-levels of the electrodes are large. Now, one may state that the LUMO-levels act as virtual states only. The inter-electrode current results from a coherent tunneling process which is induced by the so-called superexchange interelectrode coupling $[13,20,28,48]$. It drops exponentially $(\sim \exp [-(\zeta / 2)(N-1)])$ with an increase of the number of bridge units $N$. As in the theory of ET reactions in molecular complexes the sequential as well as the superexchange mechanism have also to be considered as fundamental charge transfer processes in molecular wires $[20,28,37,44,49]$.

Elastic as well as inelastic mechanisms of charge transmission through molecular wires result from a complicated interplay of dynamic and relaxation processes. For instance, the efficiency of the elastic interelectrode current strongly depends on the levelbroadening of the terminal wire units. This broadening results from the coupling to the continuous conduction band spectrum of the electrodes [13,28,30,37]. Moreover, relaxation processes within each wire determine the inelastic part of the overall current [14,28,41-43]. To achieve a correct consideration of all ET mechanisms (including the formation of superexchange couplings between the electrodes and separated wire units, as well as the various dephasing processes) a unified theoretical description becomes necessary.

It is the goal of the present paper to present such a unified description of the interelectrode current mediated by a molecular wire. However, we will concentrate on the nonadiabatic regime of charge transmission [50]. Within such a regime the ET occurs against the background of fast nuclear relaxation and, thus, the molec- ular vibrational degrees of freedom can be considered as a thermal bath. Additionally, our description focuses on a model for which the electronic energy levels of the terminal wire-units are positioned much below the electronic levels of the internal wire-units. In this case, a long-ranged superexchange ET between the terminal sites has to be considered along with the complete sequential charge transfer processes through the wire. Then, the total inelastic current is formed by a mixture of sequential and superexchange contributions.

The paper is organized as follows. Section 2 is devoted to a generalization of the spin-boson model for the description of the ET system "left electrode (L) molecular wire-right electrode (R)" (LWR). Section 3 deals with the derivation of an analytic expression for the inelastic interelectrode current. We take a particular model for the wire where the LUMO energies of its terminal units are positioned much below the LUMO energies of the internal wire-units. In Section 4, an exact analytic form of the thermally activated current is derived. The conditions are specified at which the current is given by additive contributions from the sequential (hopping) mechanism and the superexchange mechanism (related to the superexchange coupling between the terminal wire units). Analytic and numerical results on the length-dependence of the inelastic interelectrode current are presented in Section 5. In Section 6, a general discussion can be found on the length-dependence of the wire mediated current.

\section{Spin-boson model of a molecular wire}

This section is devoted to a derivation of the LWRsystem Hamiltonian valid for an arbitrary number of wire units. We will employ a semiphenomenological tight-binding approximation and suppose the electronic coupling among the wire units and between the wire and the electrode to be weak. The wire should be characterized by a linear arrangement of $N+2$ units which are embedded between two nano-electrodes (cf. Fig. 1). The energy of the transferred excess electron at the $m$ th wire unit is given by $E_{m}=E_{m}^{(0)}+\Delta E_{m}(V)$ where $\Delta E_{m}(V)$ is the shift caused by the applied voltage $V$. Obviously, the $E_{m}$ differ from the site energy $\varepsilon_{m}^{(0)}$ valid in the absence of the excess electron. Therefore, the energetic differences $\epsilon_{m}=E_{m}-\varepsilon_{m}^{(0)}=\Delta \varepsilon_{m}^{(0)}+\Delta E_{m}(V), \quad\left(\Delta \varepsilon_{m}^{(0)}=E_{m}^{(0)}-\varepsilon_{m}^{(0)}\right)$,

define the affinity of the $m$ th wire unit to an extra electron and thus can be refer to LUMO-level energies.

One way to characterize the electronic levels of the LWR-system is the use of a second-quantization notation (see, e.g. [53]) based on fermionic creation $\left(a_{j}^{+}\right)$and annihilation $\left(a_{j}\right)$ operators which act on the states $\left|N_{j}\right\rangle$. Since the occupation numbers are $N_{j}=0,1$ the states 


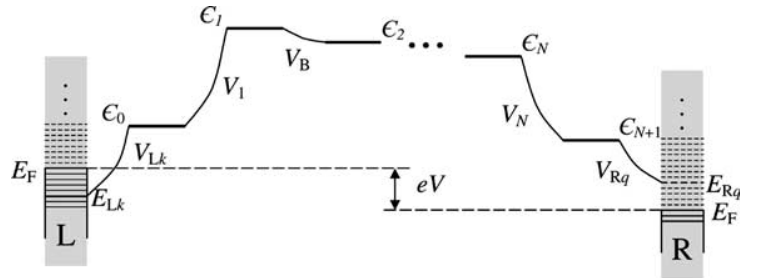

(a)

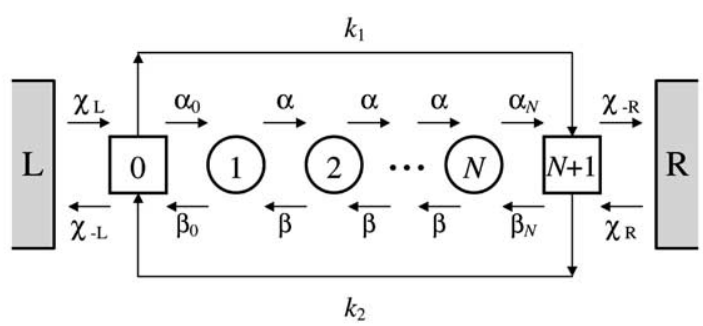

(b)

Fig. 1. Energetic (a) and kinetic scheme (b) of nonadiabatic ET through a linear molecular wire. The quantity $V_{\mathbf{L k}}\left(V_{\mathbf{R k}}\right)$ is the coupling among the LUMO-level with energy $E_{0}\left(E_{N+1}\right)$ and the electrode level with band energy $E_{\mathrm{Lk}}\left(E_{\mathrm{R} \mathbf{k}}\right)$. The coupling $V_{\mathbf{L k}}\left(V_{\mathrm{R} \mathbf{k}}\right)$ are responsible for the formation of the wire-site electrode transition rate $\chi_{\mathrm{L}}$ and $\chi_{-\mathrm{L}}\left(\chi_{\mathrm{R}}\right.$ and $\left.\chi_{-\mathrm{R}}\right)$. The wire-site to site electronic transfer couplings $V_{1} \equiv V_{10}$ $\left(V_{N} \equiv V_{N N+1}\right)$ between the (local) LUMO-levels with energies $\epsilon_{0}$ and $\epsilon_{1}$ $\left(\epsilon_{N}\right.$ and $\left.\epsilon_{N+1}\right)$ form the intersite rate constants $\alpha_{0}$ and $\alpha_{1}\left(\beta_{0}\right.$ and $\left.\beta_{1}\right)$. Analogously, the coupling $V_{\mathrm{B}} \equiv V_{m m+1}$ are responsible for the formation of wire internal interior site-site transition rates $\alpha$ and $\beta$.

$\left|0_{j}\right\rangle$ and $\left|1_{j}\right\rangle$ refer to the vacuum states of the excess electron and for the state of a single excess electron, respectively (for the present purposes there is no need to account for the electron spin). Note also the normalization condition

$\sum_{N_{j}=0,1}\left|N_{j}\right\rangle\left\langle N_{j}\right|=1$.

For the LWR-system under consideration the index $j$ has to be related to the states of the two electrodes ( $j=\mathbf{L} \mathbf{k}, \mathbf{R} \mathbf{k}$, where $\mathbf{k}$ is the wave vector of the electrode conduction band) and the local wire-unit states ( $j=m=0,1, \ldots, N+1)$. Accordingly, the electronic Hamiltonian of the LWR-system can be represented in the following form:

$H_{\mathrm{LWR}}^{(\mathrm{el})}=H_{\mathrm{LR}}^{(\mathrm{el})}+H_{\mathrm{W}}^{(\mathrm{el})}+H_{\mathrm{LR}-\mathrm{W}}^{(\mathrm{el})}$,

where the two electrode Hamiltonian read

$H_{\mathrm{LR}}^{(\mathrm{el})}=\sum_{s=\mathrm{L}, \mathrm{R}} \sum_{\mathbf{k}} E_{s \mathbf{k}} a_{s \mathbf{k}}^{+} a_{s \mathbf{k}}$.

The electronic part of the wire Hamiltonian follows as

$$
\begin{aligned}
H_{\mathrm{W}}^{(\mathrm{el})}= & \sum_{m=0}^{N+1}\left[E_{m} a_{m}^{+} a_{m}+\varepsilon_{m}^{(0)} a_{m} a_{m}^{+}\right] \\
& +\sum_{m=0}^{N}\left[V_{m m+1} a_{m}^{+} a_{m+1}+V_{m+1 m} a_{m+1}^{+} a_{m}\right] .
\end{aligned}
$$

The $V_{m m^{\prime}}$ denote the intersite electronic couplings. Finally, the wire-electrode coupling Hamiltonian is taken as

$$
\begin{aligned}
H_{\mathrm{LR}-\mathrm{W}}^{(\mathrm{el})}= & \sum_{\mathbf{k}}\left[V_{\mathrm{Lk}} a_{\mathrm{Lk}}^{+} a_{0}+V_{\mathrm{Lk}}^{*} a_{0}^{+} a_{\mathrm{Lk}}\right] \\
& +\sum_{\mathbf{q}}\left[V_{\mathrm{Rq}} a_{\mathrm{Rq}}^{+} a_{N+1}+V_{\mathrm{R} \mathbf{q}}^{*} a_{N+1}^{+} a_{\mathrm{Rq}}\right],
\end{aligned}
$$

where $V_{\mathbf{L k}}$ and $V_{\mathbf{R q}}$ are the couplings of the terminal wire units, 0 and $N+1$, to the left and to the right electrode, respectively. The electronic Hamiltonian, Eqs. (3)-(6) will be taken as a basic quantity for any further description. The expression has only to be completed by those contributions following from the nuclear dynamics.

To achieve a notation similar to that used for the standard spin-boson system we change to an electronic occupation number representation. An application of the rules $a_{j}^{+}\left|N_{j}\right\rangle=\left(1-N_{j}\right)\left|(1-N)_{j}\right\rangle$ and $a_{j}\left|N_{j}\right\rangle=N_{j} \mid$ $\left.(1-N)_{j}\right\rangle$ yields

$$
\begin{aligned}
H_{\mathrm{W}}^{(\mathrm{el})}= & \sum_{m=0}^{N+1} \sum_{N_{m}=0,1}\left[E_{m} N_{m}+\varepsilon_{m}^{(0)}\left(1-N_{m}\right)\right]\left|N_{m}\right\rangle\left\langle N_{m}\right| \\
& +\sum_{m=0}^{N}\left[V_{m m+1}\left|1_{m}\right\rangle\left\langle 0_{m}|\otimes| 0_{m+1}\right\rangle\left\langle 1_{m+1}\right|+\text { h.c. }\right] .
\end{aligned}
$$

The nuclear part of wire Hamiltonian, $H_{\mathrm{w}}^{(\mathrm{nucl})}$, will be taken in its most simple form by providing that each vibrational frequency $\omega_{\lambda m}$ with mode index $\lambda$ is independent on the presence or absence of the transferred electron at site $m$. [For the Holstein model [54] the vibrational frequencies become independent on the site index $m$ as well.] Let $M_{\lambda m}$ be the effective mass corresponding to the $\lambda$ th normal mode and let the quantities $-C_{\lambda m}$ and $+C_{\lambda m}$ define the vibrational equilibria at the presence or the absence of the excess electron, then we may write

$$
\begin{aligned}
H_{\mathrm{W}}^{(\text {nucl })}= & \sum_{m=0}^{N+1} \sum_{\lambda}\left\{\frac{p_{m \lambda}^{2}}{2 M_{m \lambda}}+\frac{M_{m \lambda} \omega_{m \lambda}^{2}}{2}\left[q_{m \lambda}-\left(2 N_{m}\right.\right.\right. \\
& \left.\left.-1) \frac{C_{m \lambda}}{M_{m \lambda} \omega_{m \lambda}^{2}}\right]^{2}\right\} \times\left|N_{m}\right\rangle\left\langle N_{m}\right| .
\end{aligned}
$$

Next, we introduce the vectorial operator $\boldsymbol{\sigma}_{m}$ which components are given by

$$
\begin{aligned}
\sigma_{m}^{z} & =\left|1_{m}\right\rangle\left\langle 1_{m}|-| 0_{m}\right\rangle\left\langle 0_{m}\right|, \\
\sigma_{m}^{x} & =\left|1_{m}\right\rangle\left\langle 0_{m}|+| 0_{m}\right\rangle\left\langle 1_{m}\right|, \\
\sigma_{m}^{y} & =-\mathrm{i}\left(\left|1_{m}\right\rangle\left\langle 0_{m}|-| 0_{m}\right\rangle\left\langle 1_{m}\right|\right) .
\end{aligned}
$$

The $\sigma_{m}^{x, y, z}$ satisfy Pauli's commutation relations and $\boldsymbol{\sigma}_{m}$ can be referred to a local Pauli pseudo-spin operator. Noting the definitions (9)-(11) as well as the normalization condition (2) one can rewrite the wire Hamiltonian $H_{\mathrm{W}}=H_{\mathrm{W}}^{(\mathrm{ell})}+H_{\mathrm{W}}^{(\text {nucl })}$ to obtain the following expression: 


$$
\begin{aligned}
H_{\mathrm{W}}= & E_{0}^{(\mathrm{el})}+E_{0}^{(\mathrm{nucl})}+(1 / 2) \sum_{m=0}^{N+1}\left[\epsilon_{m}+\sum_{\lambda} \kappa_{\lambda m}\left(b_{\lambda m}^{+}+b_{\lambda m}\right)\right] \sigma_{m}^{z} \\
& +\sum_{m=0}^{N+1} \sum_{\lambda} \hbar \omega_{\lambda m}\left(b_{\lambda m}^{+} b_{\lambda m}+1 / 2\right) \\
& +\sum_{m=0}^{N}\left[V_{m m+1} \sigma_{m}^{+} \sigma_{m+1}^{-}+V_{m+1 m} \sigma_{m+1}^{+} \sigma_{m}^{-}\right]
\end{aligned}
$$

Note the introduction of local transition operators $\sigma_{m}^{ \pm} \equiv(1 / 2)\left(\sigma_{m}^{x} \pm \mathrm{i} \sigma_{m}^{y}\right)$ as well as creation $\left(b_{\lambda m}^{+}\right)$and annihilation $\left(b_{\lambda m}\right)$ operators for the various vibrational modes. The electron-vibrational coupling at site $m$ is denoted by $\kappa_{\lambda m} \equiv C_{\lambda m} \sqrt{2 \hbar / M_{\lambda m} \omega_{\lambda m}}$. The quantities

$E_{0}^{(\mathrm{el})}=(1 / 2) \sum_{m}\left(E_{m}+\varepsilon_{m}^{(0)}\right)$

and

$E_{0}^{(\text {nucl })}=\sum_{m} \sum_{\lambda}\left(\kappa_{\lambda m}^{2} / 4 \hbar \omega_{\lambda m}\right)$,

are independent on the concrete wire state and thus do not influence the ET process. The wire Hamiltonian, Eq. (12) has to be considered as the multi-site generalization of the ordinary spin-boson Hamiltonian.

Since our approach is aimed at a description of nonadiabatic charge motion based on a fast vibrational relaxation we will consider the vibrational modes as passive bath modes and perform a polaron transformation of the wire Hamiltonian $H_{\mathrm{W}}$. Therefore, the unitary operator $\hat{S}=\prod_{m=0}^{N+1} \hat{S}_{m}$ is introduced containing the local unitary operators (see e.g. [44,54-57])

$\hat{S}_{m}=\exp \left[(1 / 2) \hat{R}_{m} \sigma_{m}^{z}\right]$,

with

$\hat{R}_{m}=\sum_{\lambda} g_{\lambda m}\left(b_{\lambda m}^{+}-b_{\lambda m}\right), \quad\left(g_{\lambda m} \equiv \kappa_{\lambda m} / \hbar \omega_{\lambda m}\right)$.

If applied to the wire Hamiltonian we obtain

$\tilde{H}_{\mathrm{W}}=\hat{S} H_{\mathrm{W}} \hat{S}^{+}=E_{0}^{(\mathrm{el})}+\tilde{H}_{\mathrm{W}}^{(\mathrm{el})}+\hat{V}_{\mathrm{W}}^{(\mathrm{tr})}+H_{\mathrm{B}}$.

The expression

$\tilde{H}_{\mathrm{W}}^{(\mathrm{el})}=(1 / 2) \sum_{m=0}^{N+1} \epsilon_{m} \sigma_{m}^{z}$,

is the electronic part of the transformed wire Hamiltonian

$H_{\mathrm{B}}=\sum_{m=0}^{N+1} \sum_{\lambda} \hbar \omega_{\lambda m}\left(b_{\lambda m}^{+} b_{\lambda m}+1 / 2\right)$,

defines the bath Hamiltonian (associated with the vibrational states of the wire and its surrounding), and

$\hat{V}_{\mathrm{W}}^{(\mathrm{tr})}=\sum_{m=0}^{N}\left[V_{m m+1} \tilde{\sigma}_{m}^{+} \tilde{\sigma}_{m+1}^{-}+V_{m+1 m} \tilde{\sigma}_{m+1}^{+} \tilde{\sigma}_{m}^{-}\right]$,

gives the modified off-diagonal interaction which is responsible for the ET processes in the wire. In the latter expression, we introduced $\tilde{\sigma}_{m}^{ \pm}=\hat{S}_{m} \sigma_{m}^{ \pm} \hat{S}_{m}^{+}=\sigma_{m}^{ \pm} e^{ \pm \hat{R}_{m}}$.

[We took into consideration the fact that $\sigma_{m}^{ \pm} \sigma_{m}^{z}-\sigma_{m}^{z} \sigma_{m}^{ \pm}= \pm 2 \sigma_{m}^{ \pm}$, and that $\left(\sigma_{m}^{z}\right)^{n}$ is equal $\sigma_{m}^{z}$ (if $n$ is the even integer) or 1 (if $n$ is the odd integer).] The wire Hamiltonian, Eq. (17) is suitable for the description of long-range ET through the wire because the off-diagonal interaction (20) includes the coupling of a particular electronic state to the bath. (Beside this property one has to bear in mind that the pseudo-spin operators $\sigma_{m}^{x, y, z}$ commutate if they refer to different sites $m$.)

Performing the polaron-transformation at the complete Hamiltonian of the LWR-system, $H_{\mathrm{LWR}}=H_{\mathrm{LWR}}^{(\mathrm{el})}+$ $H_{\mathrm{W}}^{\text {(nucl) }}$, we get

$H=\tilde{H}_{0}+V+H_{\mathrm{B}}$.

Here, $H_{\mathrm{B}}$ is given by Eq. (19), and we have

$\tilde{H}_{0}=H_{\mathrm{LR}}^{(\mathrm{el})}+\tilde{H}_{\mathrm{W}}^{(\mathrm{el})}$,

and

$V=\hat{V}_{\mathrm{W}}^{(\mathrm{tr})}+\hat{V}_{\mathrm{LR}-\mathrm{W}}^{\mathrm{tr})}$.

In both equations the operators $H_{\mathrm{LR}}^{(\mathrm{el})}, \tilde{H}_{\mathrm{W}}^{(\mathrm{el})}$ and $\hat{V}_{\mathrm{W}}^{(\mathrm{tr})}$ are defined by the Eqs. (4), (18) and (20), respectively. Furthermore, we introduced

$$
\begin{aligned}
\hat{V}_{\mathrm{LR}-\mathrm{W}}^{(\mathrm{tr})}= & \sum_{\mathbf{k}}\left[V_{\mathrm{Lk}} a_{\mathrm{Lk}}^{+} \tilde{\sigma}_{0}^{-}+V_{\mathrm{Lk}}^{*} \tilde{\sigma}_{0}^{+} a_{\mathrm{Lk}}\right] \\
& +\sum_{\mathbf{q}}\left[V_{\mathrm{Rq}} a_{\mathrm{Rq}}^{+} \tilde{\boldsymbol{\sigma}}_{N+1}^{-}+V_{\mathrm{Rq}}^{*} \tilde{\sigma}_{N+1}^{+} a_{\mathrm{Rq}}\right] .
\end{aligned}
$$

The LWR-system Hamiltonian, Eq. (22) is the one on which all further derivations of kinetic equations will be based. Note that it does not contain the nuclear energy $E_{0}^{(\text {nucl) }}$, Eq. (14) as it is the case for the nuclear Hamiltonian (12). The absence is originated by the polarontransformation which leads to a complete compensation. The wire electronic energy $E_{0}^{(\mathrm{el})}$, Eq. (13) has been omitted since it does not depend on the concrete electronic state.

\section{Formation of an interelectrode current}

Let us specialize the model of the LWR-system a little bit further. We provide for all what follows that the local LUMO-level energies $\epsilon_{m}$ of the various wire internal units $(m=1, \ldots, N)$ are position considerably above the Fermi-levels $E_{\mathrm{F}}$ of the electrodes (cf. Fig. 1(a)). And for any applied voltage considered in the following these levels should not come into resonance with the Fermi-levels. Thus, direct long-ranged resonant tunneling is of no importance [58]. In contrast, the terminal wire units $m=0$ and $m=N+1$ should have a structure different from the internal wire part (originated, for example, by absorbed atoms, by atomic clusters at the wire-electrode interface, or by additional molecular groups which belong to the terminal units [20]). As a result the LUMO- 
level energies $\epsilon_{0}$ and $\epsilon_{N+1}$ may become degenerated with the electrode's Fermi-energies at a certain voltage. Therefore, only those transition processes as represented in scheme Fig. 1(a) are assumed to contribute to the formation of the interelectrode current $I$.

To derive an expression for the current $I$ we start from the general relation

$I=-e \dot{N}_{\mathrm{L}}$,

where $e$ denotes the absolute value of the electron charge and $\dot{N}_{\mathrm{L}}=-\dot{N}_{\mathrm{R}}$ is the time-derivative of the number of those electrons moving through the molecular wire from the left $(\mathrm{L})$ to the right $(\mathrm{R})$ electrode. Since the total left electrode population reads as

$N_{\mathrm{L}}(t)=\sum_{\mathbf{k}} P_{\mathbf{L} \mathbf{k}}(t)$

we have to determine the time-derivative of the bandstate population $P_{\mathrm{Lk}}(t)$. This population is defined in the occupation number representation according to

$$
\begin{aligned}
P_{\mathbf{L k}}(t) & =\operatorname{tr}\left(\rho(t) \hat{N}_{\mathrm{Lk}}\right) \\
& =\sum_{N_{\mathbf{L k}}} \sum_{\left\{N_{s}\right\} \neq N_{\mathbf{L k}}}\left\langle N_{\mathrm{Lk}},\left\{N_{s}\right\}|\rho(t)|\left\{N_{s}\right\}, N_{\mathbf{L k}}\right\rangle N_{\mathrm{Lk}},
\end{aligned}
$$

with $\rho(t)$ denoting the electronic density matrix of the LWR-system. The electronic occupation number operator has the following form:

$\hat{N}_{\text {Lk }}=\sum_{N_{\mathbf{L k}}=0,1} N_{\text {Lk }}\left|N_{\text {Lk }}\right\rangle\left\langle N_{\text {Lk }}\right|$,

and is diagonal with respect to the occupation number states $\left|N_{\mathrm{Lk}}\right\rangle$. In the same manner one can define the electronic populations $P_{\mathrm{Rq}}(t)$ of the right electrode. The wire-site populations read

$$
\begin{aligned}
P_{m}(t) & =\operatorname{tr}\left(\rho(t) \hat{N}_{m}\right) \\
& =\sum_{N_{m}} \sum_{\left\{N_{s}\right\} \neq N_{m}}\left\langle N_{m},\left\{N_{s}\right\}|\rho(t)|\left\{N_{s}\right\}, N_{m}\right\rangle N_{m},
\end{aligned}
$$

with

$\hat{N}_{m}=\sum_{N_{m}=0,1} N_{m}\left|N_{m}\right\rangle\left\langle N_{m}\right|$,

being the site occupation number operator. Note the general notation $P_{j}(t)$ with $j=\mathbf{L k}, \mathbf{R q}, 0,1, \ldots, N+1$ which covers all populations and which can be obtained from the occupation number distribution

$P\left(N_{j}, t\right)=\sum_{\left\{N_{s}\right\} \neq N_{j}}\left\langle N_{j},\left\{N_{s}\right\}|\rho(t)|\left\{N_{s}\right\}, N_{j}\right\rangle$.

Here, the summation runs over a complete set of occupation numbers $\left\{N_{s}\right\}$ except $N_{j}$. Combining Eq. (32) with the Eqs. (30) and (31) one obtains

$$
P_{j}(t)=\sum_{N_{j}=0,1} N_{j} P\left(N_{j}, t\right),
$$

and

$1-P_{j}(t)=\sum_{N_{j}=0,1} N_{j} P\left(1-N_{j}, t\right)$,

what yields

$P_{j}(t)=P\left(1_{j}, t\right), \quad 1-P_{j}(t)=P\left(0_{j}, t\right)$.

In particular, we may write $(m=0,1, \ldots, N+1)$

$\sigma_{m}^{z}(t)=\operatorname{tr}\left(\rho(t) \sigma_{m}^{z}\right)=P\left(1_{m}, t\right)-P\left(0_{m}, t\right)=2 P_{m}(t)-1$.

Applying standard methods of nonequilibrium statistical mechanics [2,56,59-61] one may derive a closed set of kinetic equations for just all introduced populations (cf. Appendix A):

$\dot{N}_{\mathrm{L}}(t)=-\chi_{\mathrm{L}}+\Gamma_{\mathrm{L}} P_{0}(t)$,

$\dot{P}_{0}(t)=-\left(\Gamma_{\mathrm{L}}+\alpha_{0}+k_{1}\right) P_{0}(t)+\chi_{\mathrm{L}}+\beta_{0} P_{1}(t)+k_{2} P_{N+1}(t)$,

$\dot{P}_{1}(t)=-\left(\alpha+\beta_{0}\right) P_{1}(t)+\alpha_{0} P_{0}(t)+\beta P_{2}(t)$,

$\dot{P}_{m}(t)=-(\alpha+\beta) P_{m}(t)+\alpha P_{m-1}(t)+\beta P_{m+1}(t)$,

$\dot{P}_{N}(t)=-\left(\alpha_{N}+\beta\right) P_{N}(t)+\alpha P_{N-1}(t)+\beta_{N} P_{N+1}(t)$,

$\dot{P}_{N+1}(t)=-\left(\Gamma_{\mathrm{R}}+\beta_{N}+k_{2}\right) P_{N+1}(t)+\chi_{\mathrm{R}}+\alpha_{N} P_{N}(t)+k_{1} P_{0}(t)$,

$\dot{N}_{\mathrm{R}}(t)=-\chi_{\mathrm{R}}+\Gamma_{\mathrm{R}} P_{N+1}(t)$.

Here, the index $m$ runs over the wire units $2,3, \ldots$, $N-1$, only. Forward hopping rates are denoted by $\alpha_{0} \equiv k_{01}, \alpha_{N} \equiv k_{N N+1}, \alpha \equiv k_{m m+1}$ (for explanation see also Fig. 1(b)). The related backward rates are $\beta_{0} \equiv k_{01}$, $\beta_{N} \equiv k_{N+1 N}$, and $\beta \equiv k_{m+1 m}$. Since the considered rates characterize single-electron transitions between neighboring wire units the corresponding kinetic equations as well as the rate constants (describing the hopping processes within the wire) can be derived in the Born approximation with respect to the inter-site couplings $V_{m m \pm 1}$ (cf. Hamiltonian (5)). Concrete expressions for each rate are given in Eq. (A.41). There, $(\mathrm{FC})_{m s}$ denotes the Franck-Condon factor (cf. Eqs. (A.35) and (A.36)), and $V_{m s}$ is the coupling between neighboring sites ( $m$ and $s=m \pm 1$ ). Based on the same Born approximation, one can specify the rate describing the ET between each electrode and the adjacent terminal unit of the wire. In the Eq. (36) these are the electrode-wire rates $\left(\chi_{\mathrm{L}}, \chi_{\mathrm{R}}\right)$ and the wire-electrode rates $\left(\chi_{-\mathrm{L}}, \chi_{-\mathrm{R}}\right)$. Concrete expressions for these rates can be found in Eq. (A.45). The quantities

$\Gamma_{\mathrm{L}(\mathrm{R})} \equiv \chi_{\mathrm{L}(\mathrm{R})}+\chi_{-\mathrm{L}(-\mathrm{R})}$,

determine the broadening of the 0 th $((N+1)$ th) terminal electronic wire level. The broadening is caused by the interaction of these levels with the conduction band levels of the left (right) electrode as well as by the coupling to vibrational modes. If the latter coupling van- 
ishes $\Gamma_{\mathrm{L}(\mathrm{R})}$ reduces to the standard expression for electronic level broadening [28,30,37].

Along with a standard sequential site-to-site ET process the set (36) contain contributions which result from the distant superexchange coupling $V_{0 N+1}$, Eq. (A.51) between the terminal units of the wire 0 and $N+1$. There are two superexchange ET rates, the wire internal forward rate $k_{1} \equiv k_{0 N+1}$ and the backward rate $k_{2} \equiv k_{N+10}$, defined by Eq. (A.50). Details of the derivation of the basic set of coupled linear kinetic equations (36) are given in Appendix A. [Note that a linear form of set of equations is only valid for bulk electrodes where the Eq. (A.44) is fulfilled in LWR system. Besides, the population of each interior wire unit has to be small during the ET process, cf. Eq. (A.39).]

Having established the set (36) of kinetic equations we will restrict ourselves to the consideration of its stationary solution only. It allows to characterize the stationary ET regime where $\dot{P}_{m}=0$, and $\dot{N}_{\mathrm{L}}=-\dot{N}_{\mathrm{R}}=$ const. Noting the first relation the set of equations for the site populations $P_{m}$ can be solved exactly. Then, the substitution of the solution for $P_{0}$ into the first equation of the set (36) leads to the following analytic expression for the interelectrode current:

$I=e \frac{\chi_{\mathrm{L}} \Gamma_{\mathrm{R}} A_{N+1}-\chi_{\mathrm{R}} \Gamma_{\mathrm{L}} A_{0}}{\Gamma_{\mathrm{L}} A_{0}+\Gamma_{\mathrm{R}} A_{N+1}}$.

The newly introduced constants $A_{0}$ and $A_{N+1}$ read as

$$
\begin{aligned}
A_{0}= & \left(\chi_{\mathrm{L}}+\chi_{\mathrm{R}}\right)\left\{k _ { 2 } \left[\left(\alpha \alpha_{N}+\beta \beta_{0}+\alpha_{N} \beta_{0}\right) D(N-2)\right.\right. \\
& \left.\left.-\left(\alpha_{N}+\beta_{0}\right) \alpha \beta D(N-3)\right]+\beta_{0} \beta_{N} \beta^{N-1}\right\} \\
& +\chi_{\mathrm{L}} \Gamma_{\mathrm{R}}\left[\left(\alpha \alpha_{N}+\beta \beta_{0}+\alpha_{N} \beta_{0}\right) D(N-2)\right. \\
& \left.-\left(\alpha_{N}+\beta_{0}\right) \alpha \beta D(N-3)\right],
\end{aligned}
$$

and

$$
\begin{aligned}
A_{N+1}= & \left(\chi_{\mathrm{L}}+\chi_{\mathrm{R}}\right)\left\{k _ { 1 } \left[\left(\alpha \alpha_{N}+\beta \beta_{0}+\alpha_{N} \beta_{0}\right) D(N-2)\right.\right. \\
& \left.\left.-\left(\alpha_{N}+\beta_{0}\right) \alpha \beta D(N-3)\right]+\alpha_{0} \alpha_{N} \alpha^{N-1}\right\} \\
& +\chi_{\mathrm{R}} \Gamma_{\mathrm{L}}\left[\left(\alpha \alpha_{N}+\beta \beta_{0}+\alpha_{N} \beta_{0}\right) D(N-2)\right. \\
& \left.-\left(\alpha_{N}+\beta_{0}\right) \alpha \beta D(N-3)\right] .
\end{aligned}
$$

Both foregoing formulas contain the quantity

$D(M)=(\alpha \beta)^{M / 2} \frac{\sinh [\Lambda(M+1)]}{\sinh \Lambda}, \quad\left(\mathrm{e}^{-\Lambda}=(\beta / \alpha)^{1 / 2}\right)$,

which mainly determines the length-dependence of the hopping contribution to the total interelectrode current.

\section{Analytic expression for the current}

The Eqs. (38)-(40) enable us to compute the $I-V$ characteristics of the wire for different ratios among the rate constants. To get a somewhat simpler expression ready to study the wire-length dependence of the current we will specify the geometry of the wire (sites of electron localization) as well as the relations among the forward and the backward transfer rates. In the case of nonadiabatic ET under consideration the intersite electronic couplings $V_{1} \equiv V_{10}, V_{\mathrm{B}} \equiv V_{m m+1}(m=1,2, \ldots, N)$, and $V_{N} \equiv V_{N N+1} \quad$ (see Fig. 1(a)) are assumed to be small. Therefore, it is not necessary to introduce extended LUMO wire level. Just the local site energies $E_{m}$ determine the direction of the ET process. To derive a concrete expression for the energies $E_{m}$ we suppose that the transferred electron captured by a wire unit is located at the center of this unit. Furthermore, we introduce $l_{0}$ and $l_{N+1}$ as the distances between the centers of the left and the right terminal wire unit and the corresponding left and right electrode, respectively. $l_{1}$ and $l_{N}$ denote the distances between the centers of the terminal units and the adjacent right (1th) and left ( $N$ th) wire units. Finally, $l$ is the distance between the remaining internal wire centers. Then we get the voltage dependent site energies as

$$
\begin{aligned}
& E_{0}=E_{0}^{(0)}-e V\left(l_{0} / d\right), \\
& E_{N+1}=E_{N+1}^{(0)}-e V\left[1-\left(l_{N+1} / d\right)\right], \\
& E_{m}=E_{m}^{(0)}-e V(1 / d)\left[l_{0}+l_{1}+(m-1) l\right], \\
& \quad(m=1,2, \ldots, N) .
\end{aligned}
$$

Here, the distance between the two electrodes is given by

$d=l_{0}+l_{1}+(N-1) l+l_{N}+l_{N+1}$.

According to the given expressions for the site energies we remind on the detailed balance relations among the different rate expressions, which read

$\alpha_{0}=\beta_{0} \exp \left(-\Delta E_{1} / k_{\mathrm{B}} T\right)$,

$\beta_{N}=\alpha_{N} \exp \left(-\Delta E_{N} / k_{\mathrm{B}} T\right)$,

$\beta=\alpha \exp \left(-\Delta E_{m} / k_{\mathrm{B}} T\right), \quad(m=1, \ldots, N-1)$,

and

$k_{2}=k_{1} \exp \left(-\Delta E / k_{\mathrm{B}} T\right)$.

The corresponding energy gaps are defined as

$$
\begin{aligned}
& \Delta E_{1}=\left(E_{1}+\varepsilon_{0}^{(0)}\right)-\left(E_{0}+\varepsilon_{1}^{(0)}\right)=\Delta \varepsilon_{1}-e V\left(l_{1} / d\right), \\
& \left(\Delta \varepsilon_{1}=\left(E_{1}^{(0)}+\varepsilon_{0}^{(0)}\right)-\left(E_{0}^{(0)}+\varepsilon_{1}^{(0)}\right)\right), \\
& \Delta E_{N}=\left(E_{N}+\varepsilon_{N+1}^{(0)}\right)-\left(E_{N+1}+\varepsilon_{N}^{(0)}\right)=\Delta \varepsilon_{N}+e V\left(l_{N} / d\right), \\
& \left(\Delta \varepsilon_{N}=\left(E_{N}^{(0)}+\varepsilon_{N+1}^{(0)}\right)-\left(E_{N}^{(0)}+\varepsilon_{N+1}^{(0)}\right)\right), \\
& \Delta E_{m}=\left(E_{m}+\varepsilon_{m+1}^{(0)}\right)-\left(E_{m+1}+\varepsilon_{m}^{(0)}\right)=e V(l / d),
\end{aligned}
$$


and

$$
\begin{aligned}
\Delta E & =\left(E_{0}+\varepsilon_{N+1}^{(0)}\right)-\left(E_{N+1}+\varepsilon_{0}^{(0)}\right) \\
& =\Delta \varepsilon+e V\left[1-\left(l_{0}+l_{N+1}\right) / d\right], \\
(\Delta \varepsilon & \left.=\left(E_{0}^{(0)}+\varepsilon_{N+1}^{(0)}\right)-\left(E_{N+1}^{(0)}+\varepsilon_{0}^{(0)}\right)\right) .
\end{aligned}
$$

For the sake of definiteness we assumed zero voltage at the left electrode (remember $e>0$ ).

Analogous relation among the rate constants of the transitions between the electrodes and adjacent terminal wire units read as

$\chi_{\mathrm{L}(\mathrm{R})}=\chi_{-\mathrm{L}(-\mathrm{R})} \exp \left(-\Delta E_{\mathrm{L}(\mathrm{R})} / k_{\mathrm{B}} T\right)$.

The site-electrode energy gaps are

$\Delta E_{\mathrm{L}}=E_{0}-\left(\varepsilon_{0}^{(0)}+E_{\mathrm{F}}\right)=\Delta \varepsilon_{\mathrm{L}}-e V\left(l_{0} / d\right)$,

$\left(\Delta \varepsilon_{\mathrm{L}}=E_{0}^{(0)}-\left(\varepsilon_{0}^{(0)}+E_{\mathrm{F}}\right)\right)$,

and

$\Delta E_{\mathrm{R}}=E_{N+1}-\varepsilon_{0}^{(N+1)}-\left(E_{\mathrm{F}}-e V\right)=\Delta \varepsilon_{\mathrm{R}}+e V\left(l_{N+1} / d\right)$,

$$
\left(\Delta \varepsilon_{\mathrm{R}}=E_{0}^{(N+1)}-\left(\varepsilon_{0}^{(N+1)}+E_{\mathrm{F}}\right)\right) .
$$

Below we will consider the formation of an electronic current from the left to the right electrode, i.e., at $V>0$. It follows from relations (46)-(54) that

$\chi_{-\mathrm{L}} \chi_{\mathrm{R}} \beta_{0} \beta_{N} \beta^{N-1}=\chi_{-\mathrm{R}} \chi_{\mathrm{L}} \alpha_{0} \alpha_{N} \exp \left(-e V / k_{\mathrm{B}} T\right)$,

$\chi_{\mathrm{R}} \chi_{-\mathrm{L}} k_{2}=\chi_{-\mathrm{R}} \chi_{\mathrm{L}} k_{1} \exp \left(-e V / k_{\mathrm{B}} T\right)$.

Therefore, expression (38) can reduce to the more compact form

$I=e\left[1-\exp \left(-e V / k_{\mathrm{B}} T\right)\right] \frac{\chi_{\mathrm{L}}}{\Gamma_{\mathrm{L}}} \frac{F_{1}}{F_{2}}$,

where

$$
\begin{aligned}
F_{1}= & k_{1}\left(\frac{1-\gamma^{N-1}}{1-\gamma}+\frac{\alpha}{\beta_{0}}+\frac{\alpha}{\alpha_{N}} \gamma^{N-1}\right)+\frac{\alpha_{0}}{\beta_{0}} \alpha, \\
F_{2}= & \left(1+\frac{k_{1}}{\Gamma_{\mathrm{L}}}+\frac{k_{2}}{\Gamma_{\mathrm{R}}}\right)\left(\frac{1-\gamma^{N-1}}{1-\gamma}+\frac{\alpha}{\beta_{0}}+\frac{\alpha}{\alpha_{N}} \gamma^{N-1}\right) \\
& +\frac{\alpha \alpha_{0}}{\Gamma_{\mathrm{L}} \beta_{0}}+\frac{\alpha \beta_{N}}{\Gamma_{\mathrm{R}} \alpha_{N}} \gamma^{N-1} .
\end{aligned}
$$

The parameter

$\gamma \equiv \beta / \alpha=\exp \left(-e V l / d k_{\mathrm{B}} T\right)$,

characterizes the ratio between the backward and the forward wire-internal inter-site ET rate constants.

Eqs. (56)-(58) define the final analytic expression for the current. This expression has been obtained from the exact stationary solution of the set of rate equations (36) and shows a mixture of the two superexchange rate constants $k_{1}$ and $k_{2}$ and the remaining sequential rate constants. Such a mixture results in a rather complicated dependence of the current on the number $N$ of wire units. If, however, the inequalities

$k_{1}, \alpha_{0} \ll \Gamma_{\mathrm{L}}, \quad k_{2}, \beta_{N} \ll \Gamma_{\mathrm{R}}$,

are fulfilled the current (56) reduces to the sum of two separate contributions

$I=\left[1-\exp \left(-e V / k_{\mathrm{B}} T\right)\right]\left[I_{\mathrm{sup}}(N)+I_{\mathrm{seq}}(N)\right]$.

The superexchange component of the interelectrode current reads

$I_{\text {sup }}(N)=e\left(\chi_{\mathrm{L}} / \Gamma_{\mathrm{L}}\right) k_{1}$.

This expression indicates that the distant dependence of the superexchange contribution is concentrated in the rate constant $k_{1}$ defined in Eq. (A.50). Below we consider the distant behavior of the current at such voltages which do not essentially alternate the gaps (A.49). In this case the simplified form of the superexchange coupling, Eq. (A.53) is valid. It allows to reduce Eq. (62) to the expression

$I_{\text {sup }}(N)=I_{\text {sup }}^{(0)} \mathrm{e}^{-\zeta(N-1)}$,

where

$\zeta=-2 \ln \left[\left|V_{\mathrm{B}}\right| / \sqrt{\Delta E_{0} \Delta E_{N+1}}\right]$,

and

$I_{\text {sup }}^{(0)}=e\left(\chi_{\mathrm{L}} / \Gamma_{\mathrm{L}}\right) k_{1}^{(0)}$,

$k_{1}^{(0)}=\frac{2 \pi}{\hbar} \frac{\left|V_{1} V_{N+1}\right|^{2}}{\Delta E_{0} \Delta E_{N+1}}(\mathrm{FC})_{0 N+1}$.

As to the sequential component it has the form

$I_{\text {seq }}(N)=I_{\text {seq }}^{(0)}\left[1+\xi \frac{1-\gamma^{N-1}}{1-\gamma}\right]^{-1}$,

with

$I_{\text {seq }}^{(0)}=e \frac{\chi_{\mathrm{L}}}{\Gamma_{\mathrm{L}}} \frac{\alpha_{0} \alpha_{N}}{\beta_{0}+\alpha_{N}}$,

and

$\xi=\frac{1-\left(\alpha / \alpha_{N}\right)(1-\gamma)}{\left(\alpha / \beta_{0}\right)+\left(\alpha / \alpha_{N}\right)}$.

The analytic dependence of each component on the bridge unit number $N$ is provided by the diminishing parameters (64) and (69). Note that the diminishing parameter $\xi$ valid for the sequential transfer is defined through elementary rate constants. Thus, the suppression of the sequential part of the current is defined by kinetic processes. In contrast, the superexchange diminishing parameter $\zeta$ reflects the dynamic properties of the wire. 


\section{Discussion of the results}

The exact expression, Eq. (56) for the wire mediated current as well as the approximated ones, Eqs. (63) and (67) have to be considered as the main results of the present work. In particular, these formulas allow us to analyze the dependence of the current on the number of wire units, and in this way, to specify the concrete mechanism of the interelectrode current formation through the molecular wire. To present some numerical results we will use Jortner's expression for the various site-site rate constants (see Appendix A, Eqs. (A.41), (A.37) and (A.38)). They are given by

$\beta_{0}=\frac{2 \pi}{\hbar} \frac{\left|V_{1}\right|^{2}}{\hbar \omega_{1}} \Phi_{v_{1}}$

$\alpha_{N}=\frac{2 \pi}{\hbar} \frac{\left|V_{N}\right|^{2}}{\hbar \omega_{N}} \Phi_{v_{N}}$

$\alpha=\frac{2 \pi}{\hbar} \frac{\left|V_{\mathrm{B}}\right|^{2}}{\hbar \omega_{\mathrm{B}}} \Phi_{v_{\mathrm{B}}}$

$k_{1}=\frac{2 \pi}{\hbar} \frac{\left|V_{0 N+1}\right|^{2}}{\hbar \Omega} \Phi_{v}$,

where $\omega_{1}, \omega_{N}, \omega_{\mathrm{B}}$ and $\Omega$ are the characteristic vibrational frequencies related to $1 \rightarrow 0, N \rightarrow N+1$, $m \rightarrow m+1$ and $0 \rightarrow N+1$ electron transitions, respectively. The corresponding reorganization energies are denoted by $E_{1}^{(\mathrm{r})}, E_{N}^{(\mathrm{r})}, E_{\mathrm{B}}^{(\mathrm{r})}$ and $E^{(\mathrm{r})}$. In Eqs. (70)-(73), each function $\Phi_{v_{\mathrm{f}}}$ (cf. definition (A.38)) depends on two parameters: $S_{1}=E_{1}^{(\mathrm{r})} / \omega_{1}$ and $v_{1}=\Delta E_{1} / \omega_{1}$ (function $\Phi_{v_{1}}$ ), $S_{N}=E_{N}^{(\mathrm{r})} / \omega_{N}$ and $v_{N}=\Delta E_{N} / \omega_{N}$ (function $\Phi_{v_{N}}$ ), $S_{\mathrm{B}}=$ $E_{\mathrm{B}}^{(\mathrm{r})} / \omega_{\mathrm{B}}$ and $v_{\mathrm{B}}=\Delta E_{m} / \omega_{\mathrm{B}}$ (function $\Phi_{v_{\mathrm{B}}}$ ), and $S=E^{(\mathrm{r})} / \Omega$ and $v=\Delta E / \Omega$ (function $\Phi_{v}$ ). [Note that the gaps $\Delta E_{1}, \Delta E_{N}, \Delta E_{m}$ and $\Delta E$ are defined through relations (48)-(51).]

The electrode-wire transfer rates have been chosen in the form $[41,42]$

$\chi_{-\mathrm{L}(-\mathrm{R})}=\chi_{0}\left[1-n_{\mathrm{F}}\left(\Delta E_{\mathrm{L}(\mathrm{R})}\right)\right] \Phi_{0}^{(\mathrm{L}(\mathrm{R}))}$,

where $n_{\mathrm{F}}\left(\Delta E_{\mathrm{L}(\mathrm{R})}\right)=\left[\exp \left(\Delta E_{\mathrm{L}(\mathrm{R})} / k_{\mathrm{B}} T\right)+1\right]^{-1}$ is the Fermi distribution function with the energy gaps defined in the Eq. (53) as well as (54), and we introduced

$\Phi_{0}^{(\mathrm{L})}=\mathrm{e}^{-S_{0} \operatorname{coth}\left(\hbar \omega_{0} / k_{\mathrm{B}} T\right)} I_{0}\left(2 S_{0}\left[n\left(\omega_{0}\right)\left(1+n\left(\omega_{0}\right)\right)\right]^{1 / 2}\right)$.

[The quantity $\Phi_{0}^{(\mathrm{R})}$ follows from $\Phi_{0}^{(\mathrm{L})}$ by replacing $S_{0}=E_{0}^{(\mathrm{r})} / \omega_{0}$ and $\omega_{0}$ by $S_{N+1}=E_{N+1}^{(\mathrm{r})} / \omega_{N+1}$ and $\omega_{N+1}$, respectively.] In Eq. (74), $\chi_{0}$ is the width of a single electronic level caused by the interaction with one of the two electrodes in the absence of electron-vibration coupling. This conclusion is based on the definition of the level broadenings $\Gamma_{\mathrm{L}(\mathrm{R})}$, Eq. (37). Actually, for a symmetric wire and in the absence of electron-vibration coupling where $\Phi_{0}^{(\mathrm{L}(\mathrm{R}))}$ equals one, it follows $\Gamma_{\mathrm{L}}=\Gamma_{\mathrm{R}}=$ $\chi_{0}$.

Fig. 2 demonstrates the agreement between the exact and the approximate description of the current, thus supporting the conclusion that the additive form of the current, Eq. (61) is sufficiently correct if the inequalities (60) are fulfilled. If this is not the case, there is a difference in the wire-length dependence of the current, Fig. 3. This difference is more significant for a small number of wire units at which the influence of the superexchange ET channel is larger. Fig. 4 shows that the superexchange channel becomes dominant if the current is measured at lower temperature. Note, however, that the drop of the superexchange component does not follow the conventional dependence $I_{\text {sup }} \sim$ $\exp [-\zeta(N-1)]$ (see the curve at $T=180 \mathrm{~K}$ ). This is due to the fact that Eq. (63) contains an additional weak dependence on $N$ via $I_{\text {sup }}^{(0)}$ Eq. (65). Indeed, $I_{\text {sup }}^{(0)}$ is proportional to the rate $k_{1}^{(0)}$, Eq. (66) which in turn is proportional to the Franck-Condon factor $(\mathrm{FC})_{0 N+1}$. This Franck-Condon factor depends on the driving force $\Delta E=\epsilon_{0}-\epsilon_{N+1}=\Delta \epsilon^{(0)}-e V\left[l_{1}+l_{N}+l(N-1)\right]$, Eq. (51) which for $V \neq 0$ is a function of the number of wire units connecting the sites 0 and $N+1$. Therefore, we may conclude that the superexchange part of the current results in a slightly corrected exponential decrease with increasing $N$, while the sequential part shows a smooth decrease.

The $N$-dependence of $I_{\text {seq }}$ is contained in the factor $\left(1-\gamma^{N-1}\right) /(1-\gamma)$ which has the two limits, $N-1$ (at

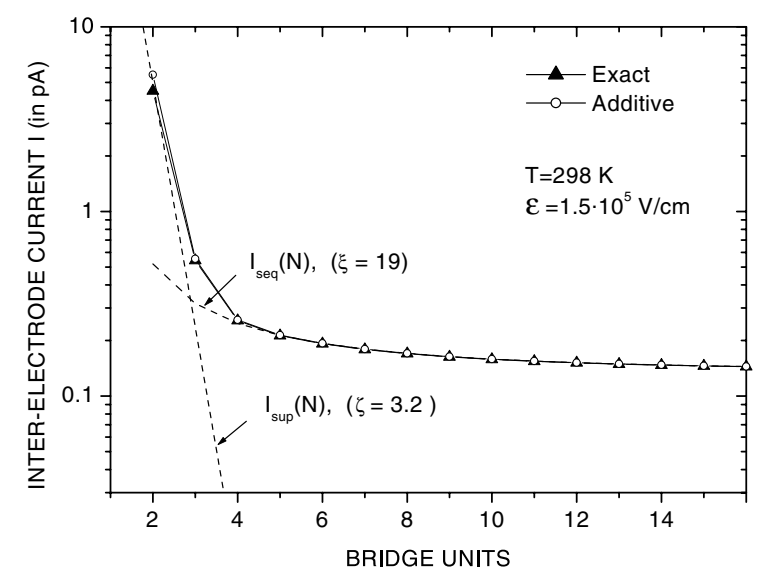

Fig. 2. Length-dependence of the interelectrode current at a fixed strength of the applied electric field $\mathscr{E}=V / d=1.5 \times 10^{5} \mathrm{~V} / \mathrm{cm}$. The description based on the exact expression, Eq. (56), and the approximate one, Eq. (61) coincide. As a consequence, the current can be represented in this case as an additive contribution of the superexchange ET mechanisms and the sequential mechanism. The rate constants are defined by Eqs. (70)-(74) with the parameters $\Delta \varepsilon_{0}=$ $\Delta \varepsilon_{N+1}=0.1 \mathrm{eV}, \Delta \varepsilon_{1}=\Delta \varepsilon_{N}=0.25 \mathrm{eV}, \Delta \varepsilon=0, E_{0}^{(\mathrm{r})}=E_{N+1}^{(\mathrm{r})}=0.7 \mathrm{eV}$, $E_{1}^{(\mathrm{r})}=E_{N}^{(\mathrm{r})}=0.4 \mathrm{eV}, E_{\mathrm{B}}^{(\mathrm{r})}=0.6 \mathrm{eV}, E^{(\mathrm{r})}=0.2 \mathrm{eV}, V_{1}=V_{N}=0.02 \mathrm{eV}$, $V_{\mathrm{B}}=0.03 \mathrm{eV}, \omega_{0}=\omega_{N+1}=800 \mathrm{~cm}^{-1}, \omega_{1}=\omega_{N}=\omega_{\mathrm{B}}=\Omega=50 \mathrm{~cm}^{-1}$, $\chi_{0}=10^{13} \mathrm{~s}^{-1}, l_{0}=l_{N+1}=4 \AA, l_{1}=l_{N}=l=3 \AA$. 


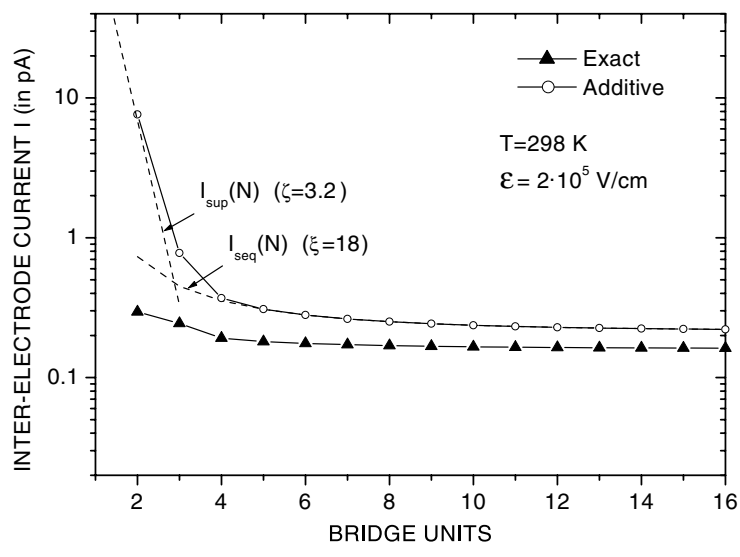

Fig. 3. Length-dependence of the interelectrode current at a fixed strength of the applied electric field $\mathscr{E}=V / d=2 \times 10^{5} \mathrm{~V} / \mathrm{cm}$. There occurs a distinct difference between the exact expression, Eq. (56) and the approximate theory, see Eq. (61). The set of parameters are the same as in Fig. 2 except $\omega_{0}=\omega_{N+1}=50 \mathrm{~cm}^{-1}, \omega_{1}=\omega_{N}=\omega_{\mathrm{B}}=$ $\Omega=100 \mathrm{~cm}^{-1}$.

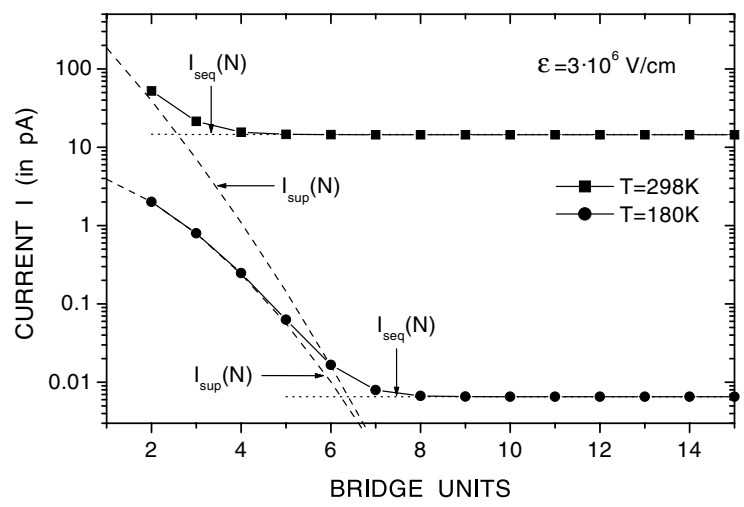

Fig. 4. Length-dependence of the interelectrode current at a fixed strength of the applied electric field $\mathscr{E}=V / d=3 \times 10^{6} \mathrm{~V} / \mathrm{cm}$ and for two different temperatures at $T=298 \mathrm{~K}$ and at $T=180 \mathrm{~K}$. The description is based on the exact result (56). The approximate description using Eq. (61), is depicted by dashed lines. The deviation from an exponential decrease of the superexchange component of the current can be clearly identified. The rate constants are defined according to the Eqs. (70)-(74) with parameters $\Delta \varepsilon_{0}=\Delta \varepsilon_{N+1}=0.1 \mathrm{eV}, \Delta \varepsilon_{1}=$ $\Delta \varepsilon_{N}=0.4 \mathrm{eV}, \Delta \varepsilon=0, \quad E_{0}^{(\mathrm{r})}=E_{N+1}^{(\mathrm{r})}=0.7 \mathrm{eV}, \quad E_{1}^{(\mathrm{r})}=E_{N}^{(\mathrm{r})}=0.8 \mathrm{eV}$, $E_{\mathrm{B}}^{(\mathrm{r})}=0.6 \quad \mathrm{eV}, \quad E^{(\mathrm{r})}=1 \quad \mathrm{eV}, \quad V_{1}=V_{N}=0.05 \quad \mathrm{eV}, \quad V_{\mathrm{B}}=0.07 \quad \mathrm{eV}$, $\omega_{0}=\omega_{N+1}=800 \mathrm{~cm}^{-1}, \omega_{1}=\omega_{N}=\omega_{\mathrm{B}}=\Omega=50 \mathrm{~cm}^{-1}, \chi_{0}=10^{13} \mathrm{~s}^{-1}$, $l_{0}=l_{N+1}=4 \AA, l_{1}=l_{N}=l=3 \AA$.

$\gamma \approx 1$ ), and 1 (at $\gamma \ll 1$ ). Therefore, the sequential contribution to the total current shows to limiting cases

$I_{\mathrm{seq}}(N)=\frac{I_{\mathrm{seq}}^{(0)}}{1+\xi(N-1)}$,

$\xi=\frac{1}{\left(\alpha / \beta_{0}\right)+\left(\alpha / \alpha_{N}\right)}, \quad(\beta \approx \alpha)$,

and

$I_{\mathrm{seq}}(N)=\frac{I_{\mathrm{seq}}^{(0)}}{1+\xi}$, $\xi=\frac{1-\left(\alpha / \alpha_{N}\right)}{\left(\alpha / \beta_{0}\right)+\left(\alpha / \alpha_{N}\right)}, \quad(\beta \ll \alpha)$.

In the first limit, Eq. (76), $I_{\text {seq }}(N)$ shows a hyperbolic decrease with increasing $N$. Such a behavior of the current is caused by the presence of hopping processes for which the forward and backward wire-internal intersite rate constants are equal one to another. Physically, this case corresponds to a small intersite voltage bias so that $\exp \left(-e V l / d k_{\mathrm{B}} T\right) \approx 1$. The second limit, Eq. (77), is realized for a large intersite voltage bias when $\exp \left(-e V l / d k_{\mathrm{B}} T\right) \ll 1$. Here, the ET along the regular part of the wire is exclusively defined by the forward rate constant $\alpha$. It corresponds to a directed ET in the wire so that the interelectrode current does not depend on the number of wire units. Both limiting cases clearly indicate that at a large number of wire units the sequential mechanism prevails the superexchange one. Such a conclusion follows from the additive form of the current, Eq. (61) and additionally, if the conditions for deep tunneling are fulfilled. As a matter of fact, the additive form of the current is obtained if, and only if, the inequalities (60) are fulfilled in the course of the ET. Furthermore, in this case all site populations $P_{m}$ related to the internal wire units $m=1,2, \ldots, N$ become small.

Note that above mentioned $N$-dependence of the sequential contribution to the total current is only valid if this dependence is studied at a fixed strength of the applied electric field $\mathscr{E}=V / d$. Just in this case the ratio (59) becomes a length-independent parameter. Thus the length dependence of the hopping component of the current is only given by the factor $\gamma^{N-1}$ (see Eqs. (56)(58) as well as Eqs. (61) and (67)). Such a result does not remain valid if the $N$-dependence is studied for a fixed voltage $V$ (see also the discussion in [62]). Fig. 5

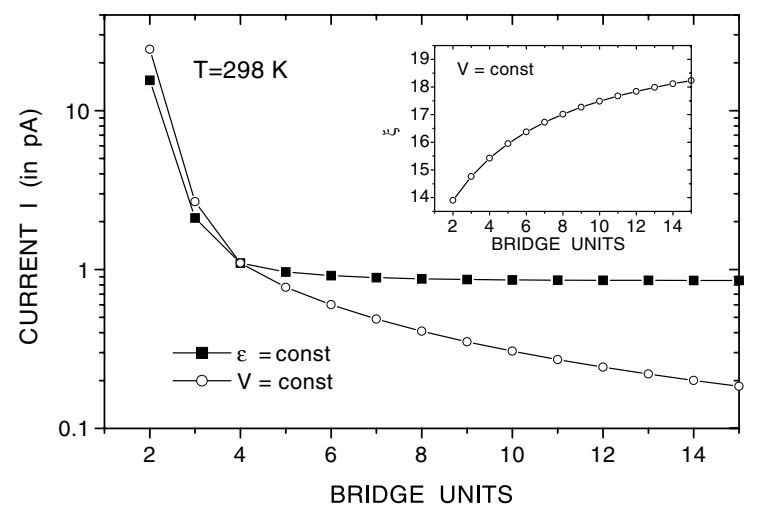

Fig. 5. Length-dependence of the interelectrode current at a fixed strength of the applied electric field $\mathscr{E}=V / d=4.35 \times 10^{5} \mathrm{~V} / \mathrm{cm}$ and at a voltage $V=0.9 \mathrm{~V}$. There occurs a strong difference in the wire-length dependence which is caused by the contribution corresponding the sequential ET. The insert depicts the variation of the sequential diminishing parameter $\zeta$ as a function of the wire length. The description is based on the exact Eq. (56). The parameters are the same as those for Fig. 2. 
illustrates this statement. If the $N$-dependence is studied at $V=$ const., the sequential diminishing parameter $\xi$ exhibits an additional $N$-dependence (see inset in Fig. 5). It follows from the ratio (59) of the backward and the forward intersite rate constants and by the electrodeelectrode distance $d$, Eq. (45).

\section{Conclusions}

In the present paper, we have generalized the spinboson model to a description of nonadiabatic electron transfer (ET) processes which take place in systems with an arbitrary number of localization sites of the excess electron. Site pseudo-spin operators (9)-(11) have been introduced and a Hamiltonian (22) has been presented which describes the ET in the system "left (L) electrode-molecular wire-right (R) electrode". The linear coupling of the excess electron levels to nuclear vibrations was taken into account in an exact manner by carrying out the well-known polaron transformation (15). After such a transformation the coupling among the electronic levels is combined with a coupling to the nuclear vibrations, Eq. (24), and in turn becomes a weak perturbation. The used local pseudo-spin operators act in the space of occupation number states. Correspondingly, we have derived a master equation for the occupation number distribution, Eq. (A.28). This equation presents a key-result for the unified description of charge motion through a molecular wire. We arrived at a set of kinetic equations for state populations including corresponding sequential and superexchange ET rate constants. It is of prominent importance to utilize the occupation number representation, since it easily allows to consider Pauli's exclusions principle which results in a nonlinearity in the kinetic equations (A.34) and (A.42).

The generalization of the spin-boson model to the description of charge motion through molecular wires has been done for the case of thermally activated interelectrode transfer. In this case, the transferred electron is released from the electrode to the adjacent terminal unit of the wire. Afterwards the electron either moves through the wire units (the sequential pathway) or it performs a single transition to the terminal group at the other tail of the wire (the superexchange pathway). A particular model has been considered for which the wire-internal units $1,2, \ldots, N$ (cf. the scheme in Fig. 1) act as a bridging molecular structure between the terminal units. This model also ensures that these units are only weakly populated by the transferred electron. Resulting from this we may utilize a linear version of the kinetic equations, Eq. (36). In this connection it is important that we have been able to derive the exact solution of these kinetic equations and thus to have at hand an analytic form for the interelectrode current
(Eqs. (38)-(41)). Taking the exact (Eqs. (56)-(58)) as well as approximated analytic expressions for the current (Eqs. (61)-(67)) we have analyzed its length-dependence at a fixed electric field $\mathscr{E}=V / d$ as well as at a fixed voltage $V$. Such an analysis of the wire-length dependence of the current allowed us to draw the following conclusions.

(1) The general case of interelectrode current formation there is a strong mixing between the sequential and the superexchange mechanism, note the exact expression for the current, Eq. (56). An naive, additive contribution of both mechanisms, see Eq. (61) only becomes justified if the specific conditions, Eq. (60) are fulfilled. Physically, these conditions correspond to a fast ET between the electrodes and the adjacent wire units. (Then, an ET is defined by the integral level broadenings $\Gamma_{\mathrm{L}}$ and $\Gamma_{\mathrm{R}}$, Eq. (37).)

(2) If $V>V_{\text {res }}$, where $V_{\text {res }}$ is defined by the gap between the terminal LUMO-level energy $\epsilon_{0}$, Eq. (1) and the Fermi-level $E_{\mathrm{F}}$, a resonant ET from electrode $\mathrm{L}$ to the terminal wire unit 0 occurs. Furthermore, the siteelectrode transition rate $\chi_{-\mathrm{L}}$ becomes small compared to the electrode-site rate $\chi_{\mathrm{L}}$, so that $\Gamma_{\mathrm{L}}$ starts to coincide with $\chi_{\mathrm{L}}$. This means that a release of an electron from electrode $\mathrm{L}$ to the terminal wire unit 0 does not require thermal activation. Therefore, the part of the current based on the superexchange ET between the terminal wire units is also present at low temperatures. This fact is illustrated by Eqs. (65) and (66), and Fig. 4.

(3) There exists a distinct difference in the wire-length dependence of the current found at a fixed electric field $\mathscr{E}=V / d$ and at a fixed voltage $V$ (cf. Fig. 5). This difference is caused by several factors. First of all, if $\mathscr{E}=V / d=$ const. the parameter $\gamma$, Eq. (59) as well as the rate constants $\alpha, \beta, \alpha_{0(N)}$ and $\beta_{0(N)}$ (defining the sequential ET channel) become wire-length independent quantities. Therefore, the $N$-dependence of the sequential component of the current is completely defined by the factor $\gamma^{N-1}$ (or/and $\left(1-\gamma^{N-1}\right) /(1-\gamma)$ ). In contrast, the superexchange site-site coupling defined by Eq. (A.51) includes an $\mathrm{N}$-dependence only via the number of inter-wire transfer couplings, thus it can be approximated by the simplified expression (A.53). The latter contains the diminishing parameter $\zeta$, Eq. (64), which does not depend on $N$. Therefore, at $\mathscr{E}=V / d=$ const. the superexchange component of the current shows a weak deviation from an exponential law $\sim \exp [-\zeta(N-$ 1)] caused by the dependence of the driving force $\Delta E$, Eq. (51) on $N$. The length-dependence of the current becomes more complicated if the current is measured at $V=$ const. In this case all rate constants depend on $N$ due to the dependence of the driving forces, which all belong to separate ET reactions, Eqs. (48)-(51) on the distance $d$. Additionally, the ratio (59) and the superexchange coupling (A.51) also show the $N$-dependence. Thus, the length-dependence of the current is much 
more complex as compared to the case of bridge-mediated donor-acceptor ET [52].

\section{Acknowledgements}

The work was supported in part by the NASU scientific program on Nanophysics and Nanoelectronics (Project N 0102U002329, E.P.), the Volkswagen-Stiftung, Grant No. I/78117 within the priority area "Intraand Intermolecular Electron Transfer" (E.P. and V.M.), the Volkswagen-Stiftung, Grant No. I77217 within the priority area "Single Molecules" (P.H.) and by the Sonderforschungsbereich SFB 486 of the Deutsche Forschungsgemeinschaft.

\section{Appendix A. Master equation in the occupation number representation and kinetic equations}

In the case of the considered nonadiabatic ET the transition processes take place on a time scale $\Delta t$ which significantly exceeds the characteristic time $\tau_{\text {rel }}$ of vibrational relaxation. Following from this the vibrational degrees of freedom can be considered as a thermal bath. Moreover, the considered regime of ET can be characterized by a certain property of the total density operator $\rho^{(S-B)}(t)$ of the LWR-system (including the vibrational degrees of freedom). This property is related to the part $\rho_{\mathrm{d}}^{(S-B)}(t)$ of $\rho^{(S-B)}(t)$ which is diagonal in a representation based on the used local electronic states and the related vibrational states. We have

$\lim _{t \gg \tau_{\mathrm{rel}}} \rho_{\mathrm{d}}^{(S-B)}(t)=W_{\mathrm{B}} \rho_{\mathrm{d}}(t)$,

where $\rho_{\mathrm{d}}(t)$ is the diagonal part of the electronic density operator (taken in the mentioned basis). The latter is defined according to the standard expression

$\rho(t)=\operatorname{tr}_{\mathrm{vib}} \rho^{(S-B)}(t)$,

where $\operatorname{tr}_{\mathrm{vib}}$ denotes the trace with respect to all vibrational states. Finally, we also introduced

$W_{\mathrm{B}}=\exp \left(-H_{\mathrm{B}} / k_{\mathrm{B}} T\right) / \operatorname{tr}_{\mathrm{vib}} \exp \left(-H_{\mathrm{B}} / k_{\mathrm{B}} T\right)$,

which is the equilibrium vibrational (bath) density operator. The factorization ansatz (A.1) corresponds to a basic assumption of nonequilibrium statistical mechanics (see, e.g. $[2,56,59,61])$. If $\hat{O}$ is an electronic operator which is diagonal in the mentioned basis then the corresponding average can be derived according to

$\bar{O}(t)=\operatorname{tr}(\rho(t) \hat{O})=\operatorname{tr}\left(\rho_{\mathrm{d}}(t) \hat{O}\right)$.

Consequently, only the diagonal part of the electronic density operator $\rho_{\mathrm{d}}(t)$ is important for the determination of $\bar{O}(t)$. Indeed, this is the case when evaluating the electronic state populations $P_{\mathbf{L k}}(t)$ and $P_{\mathbf{R q}}(t)$ for the electrodes as well as the site populations $P\left(N_{m}, t\right)$ for the wire units $m=0,1, \ldots, N+1$ (see definition (32)).
Our next aim will be the derivation of kinetic equations for the population number distributions $P\left(N_{j}, t\right)$ $(j=\mathbf{L} \mathbf{k}, \mathbf{R q}, 0,1, \ldots, N+1)$. In doing this we note that the occupation number operators $\hat{N}_{j}$ replace the diagonal operator $\hat{O}$ introduced so far. The eigenstates of the LWR system are defined by the set of occupation numbers $\left\{N_{j}=0,1\right\}$ and read

$|a\rangle \equiv\left|\left\{N_{j}\right\}\right\rangle=\prod_{\mathbf{k}}\left|N_{\mathrm{Lk}}\right\rangle \prod_{\mathbf{q}}\left|N_{\mathrm{Rq}}\right\rangle \prod_{m=0}^{N+1}\left|N_{m}\right\rangle$.

Therefore, we get

$$
\begin{aligned}
P\left(N_{\mathrm{Lk}}, t\right) & =\sum_{a \neq N_{\mathbf{L k}}} \rho_{a a}(t) \\
& =\sum_{\left\{N_{s}\right\} \neq N_{\mathbf{L k}}}\left\langle N_{\mathrm{Lk}},\left\{N_{s}\right\}|\rho(t)|\left\{N_{s}\right\}, N_{\mathrm{Lk}}\right\rangle,
\end{aligned}
$$

where the summation covers all occupation numbers $\left\{N_{j}\right\}$ except $N_{\text {Lk }}$. In an analogous way we obtain for the distribution function of the wire-site populations

$$
\begin{aligned}
P\left(N_{m}, t\right) & =\sum_{a \neq N_{m}} \rho_{a a}(t) \\
& =\sum_{\left\{N_{s}\right\} \neq N_{m}}\left\langle N_{m},\left\{N_{s}\right\}|\rho(t)|\left\{N_{s}\right\}, N_{m}\right\rangle .
\end{aligned}
$$

Denoting the diagonal elements of the electronic density matrix (in the occupation number representation) as

$\mathscr{P}_{a}(t) \equiv \rho_{a a}(t)=\langle a|\rho(t)| a\rangle=\left\langle a\left|\rho_{\mathrm{d}}(t)\right| a\right\rangle$,

then it becomes obvious that

$P\left(N_{j}, t\right)=\sum_{a \neq N_{j}} \mathscr{P}_{a}(t), \quad(j=\mathrm{L} \mathbf{k}, \mathbf{R} \mathbf{q}, 0,1, \ldots, N+1)$.

Therefore, the derivation of kinetic equations for the populations $P\left(N_{j}, t\right)$ may be based on the master equation for $\mathscr{P}_{a}(t)$ or, what is equivalent, on the master equation for the diagonal density operator $\rho_{\mathrm{d}}(t)$.

\section{A.1. Master equation for the diagonal components of the density matrix of an open quantum system}

Following the approach which leads to the wellknown Nakajima-Zwanzig equation (see details in [61]) we introduce the projection operators $\hat{T}_{d}$ and $\hat{T}_{n d}=$ $1-\hat{T}_{d}$. They separate any operator $A=\sum_{a b} A_{a b}|a\rangle\langle b|$ expanded with respect to the basis states defined in Eq. (A.5) into diagonal and off-diagonal components:

$$
\begin{aligned}
& \hat{T}_{d} A=A_{d}=\sum_{a} A_{a a}|a\rangle\langle a|, \\
& \hat{T}_{n d} A=A_{n d}=\sum_{a b}\left(1-\delta_{a b}\right) A_{a b}|a\rangle\langle b| .
\end{aligned}
$$

Of course, the definition of the projection operators becomes unambiguously only since we related the definition to a particular basis set. 
In the following we will identify the arbitrary operator $A$ with $\rho^{(S-B)}(t)$ which is governed by the Liouvillevon Neumann equation

$\dot{\rho}^{(S-B)}(t)=-\mathrm{i} \mathscr{L} \rho^{(S-B)}(t)$,

$\mathscr{L} \equiv(1 / \hbar)[H, \ldots]$ is the Liouville superoperator specified by the total Hamiltonian $H=H_{0}+V$. According to this separation we may write $\mathscr{L}=\mathscr{L}_{0}+\mathscr{L}_{V}$ with $\mathscr{L}_{0} \equiv(1 / \hbar)\left[H_{0}, \ldots\right] \mathscr{L}_{V} \equiv(1 / \hbar)[V, \ldots]$. In the studied case of the LWR-system the Hamiltonian $H$ is given by Eq. (22) and thus we may identify

$H_{0}=\tilde{H}_{0}+H_{\mathrm{B}}$,

with $\tilde{H}_{0}$ and $H_{\mathrm{B}}$ given in the Eqs. (23) and (19), respectively. Moreover, the interaction $V$ is defined by Eq. (24) together with the Eqs. (20) and (25).

Utilizing the initial condition in the standard form $\rho_{n d}^{(S-B)}(0)=0$ (decoupling of the system from the bath) and bearing in mind the properties

$\hat{T}_{d} \mathscr{L}_{0} \rho^{(S-B)}(t)=0$,

$\hat{T}_{n d} \mathscr{L}_{0} \rho_{\mathrm{d}}^{(S-B)}(t)=0$,

$\hat{T}_{n d} \mathscr{L}_{0} \rho^{(S-B)}(t)=\mathscr{L}_{0} \rho_{n d}^{(S-B)}(t)$,

$\hat{T}_{d} \mathscr{L}_{V} \rho^{(S-B)}(t)=\hat{T}_{d} \mathscr{L}_{V} \rho_{\text {nd }}^{(S-B)}(t)$,

we may reduce Eq. (A.11) to

$\dot{\rho}_{d}^{(S-B)}(t)=-\mathrm{i} \hat{T}_{d} \mathscr{L}_{V} \rho_{n d}^{(S-B)}(t)$,

where the off-diagonal component is coupled to the diagonal one by the relation

$\rho_{n d}^{(S-B)}(t)=-\mathrm{i} \int_{0}^{t} \mathrm{~d} \tau \mathscr{U}(\tau) \hat{T}_{d} \mathscr{L}_{V} \rho_{\mathrm{d}}^{(S-B)}(t-\tau)$.

The unitary time-propagation superoperator reads

$\mathscr{U}(\tau)=\exp \left[-\mathrm{i}\left(\mathscr{L}_{0}+\hat{T}_{n d} \mathscr{L}_{V}\right) \tau\right]$.

In the case of the considered nonadiabatic ET memory effects are of less importance. We replace in Eq. (A.18) $\rho_{\mathrm{d}}^{(S-B)}(t-\tau)$ by $\rho_{\mathrm{d}}^{(S-B)}(t)$ and extend the time-integral up to infinity. Then, employing relation (A.18) and the asymptotic property (A.1) we end up with the following master-equation:

$\dot{\rho}_{d}(t)=-\mathscr{R} \rho_{\mathrm{d}}(t)$,

where

$\mathscr{R}=\int_{0}^{\infty} \mathrm{d} \tau \operatorname{tr}_{\text {vib }}\left\{\hat{T}_{d} \mathscr{L}_{V} \mathscr{U}(\tau) \hat{T}_{n d} \mathscr{L}_{V} W_{\mathrm{B}}\right\}$,

is the superoperator which determines the transfer processes.

In order to compute $\mathscr{R}$ we expand $\int_{0}^{\infty} \mathrm{d} \tau \mathscr{U}(\tau)$ with respect to $\mathscr{L}_{V}$. Defining the superoperator $\mathscr{U}^{(0)}(\tau)=$ $\exp \left(-\mathrm{i} \mathscr{L}_{0} \tau\right)$ and noting

$$
\begin{aligned}
\int_{0}^{\infty} \mathrm{d} \tau \mathrm{e}^{\mathrm{i} \omega \tau} \mathscr{U}(\tau) & =\mathrm{i} \lim _{\varepsilon \rightarrow+0}\left(\omega-\mathscr{L}_{0}-\hat{T}_{n d} \mathscr{L}_{V}+\mathrm{i} \varepsilon\right)^{-1} \\
\equiv \mathrm{i} \mathscr{G}(\omega), & \\
\int_{0}^{\infty} \mathrm{d} \tau \mathrm{e}^{\mathrm{i} \omega \tau} \mathscr{U}^{(0)}(\tau) & =\mathrm{i} \lim _{\varepsilon \rightarrow+0}\left(\omega-\mathscr{L}_{0}+\mathrm{i} \varepsilon\right)^{-1} \\
& \equiv \mathrm{i} \mathscr{G}^{(0)}(\omega),
\end{aligned}
$$

one can see that [61]

$$
\begin{aligned}
\mathscr{G}(\omega)= & \mathscr{G}^{(0)}(\omega)+\mathscr{G}^{(0)}(\omega) \hat{T}_{n d} \mathscr{L}_{V} \mathscr{G}^{(0)}(\omega) \\
& +\mathscr{G}^{(0)}(\omega) \hat{T}_{n d} \mathscr{L}_{V} \mathscr{G}^{(0)}(\omega) \hat{T}_{n d} \mathscr{L}_{V} \mathscr{G}^{(0)}(\omega) \\
& +\cdots
\end{aligned}
$$

Since $\mathscr{G}^{(0)}(0)=\int_{0}^{\infty} \mathrm{d} \tau \mathscr{U}^{(0)}(\tau)$, we get the expansion

$$
\begin{aligned}
\int_{0}^{\infty} \mathrm{d} \tau \mathscr{U}(\tau)= & \int_{0}^{\infty} \mathrm{d} \tau \mathscr{U}^{(0)}(\tau)+\int_{0}^{\infty} \mathrm{d} \tau \mathscr{U}^{(1)}(\tau) \\
& +\int_{0}^{\infty} \mathrm{d} \tau \mathscr{U}^{(2)}(\tau)+\cdots, \\
\int_{0}^{\infty} \mathrm{d} \tau \mathscr{U}^{(n)}(\tau)= & (-\mathrm{i})^{n} \int_{0}^{\infty} \mathrm{d} \tau \int_{0}^{\infty} \mathrm{d} \tau_{1} \int_{0}^{\infty} \mathrm{d} \tau_{2} \cdots \\
& \times \int_{0}^{\infty} \mathrm{d} \tau_{n} \mathscr{U}^{(0)}(\tau) \hat{T}_{n d} \mathscr{L}_{V} \mathscr{U}^{(0)}\left(\tau_{1}\right) \\
& \times \hat{T}_{n d} \mathscr{L}_{V} \mathscr{U}^{(0)}\left(\tau_{2}\right) \cdots \hat{T}_{n d} \mathscr{L}_{V} \mathscr{U}^{(0)}\left(\tau_{n}\right) .
\end{aligned}
$$

\section{A.2. Kinetic equations for occupation number populations}

Let us start from the following linear balance equations:

$\dot{\mathscr{P}}_{a}(t)=-\sum_{b}\langle a|(\mathscr{R}|b\rangle\langle b|)| a\rangle \mathscr{P}_{b}(t)$,

for the total electronic populations $\mathscr{P}_{a}(t)$ of the LWRsystem. [In line with definition (A.8) these equations are completely identical to master Eq. (A.20).] Taking into account the fact that for the occupation number representation the many-particle state (A.5) appears as a product of single-particle states $\left|N_{j}\right\rangle$, it is convenient to denote the many-particle population $\mathscr{P}_{a}(t)=\mathscr{P}_{\left\{N_{j}\right\}}(t)$ as a product of single-particle populations

$\mathscr{P}_{\left\{N_{j}\right\}}(t)=\prod_{\mathbf{k}} P\left(N_{\mathrm{Lk}}, t\right) \prod_{\mathbf{q}} P\left(N_{\mathrm{R} \mathbf{q}}, t\right) \prod_{m=0}^{N+1} P\left(N_{m}, t\right)$.

Introducing this ansatz into Eq. (A.26), summarizing both parts of Eq. (A.26) over all occupation numbers except $N_{j}$, and utilizing the definition Eq. (A.9) we arrive at the following kinetic equations for the occupation number distributions $P\left(N_{j}, t\right)$ 


$$
\begin{aligned}
\dot{P}\left(N_{j}, t\right)= & \sum_{\left\{N_{j}^{\prime \prime}\right\} \neq N_{j}} \sum_{\left\{N_{j}^{\prime}\right\}}\left\langle\left\{N_{j}^{\prime \prime}\right\}\left|\left(\mathscr{R}\left|\left\{N_{j}^{\prime}\right\}\right\rangle\left\langle\left\{N_{j}^{\prime}\right\}\right|\right)\right|\left\{N_{j}^{\prime \prime}\right\}\right\rangle \\
& \times \mathscr{P}_{\left\{N_{j}^{\prime}\right\}}(t) .
\end{aligned}
$$

These equations represent master equations written in the occupation number representation. They are of basic importance for the derivation of kinetic equations for the occupation number distributions $P\left(N_{j}, t\right)$ and for the state populations $P_{j}(t)$. To write down the set of equations (A.28) in a more concrete form one can utilize the expansion Eq. (A.25) and the expression for the Hamiltonian $H_{0}$, Eqs. (A.12), (23) and (19), as well as the interaction $V$, Eq. (24). Moreover, one also has to take into consideration the following rules specifying the action of the pseudo-spin operators on the occupation number states

$\sigma_{m}^{z}\left|N_{m}\right\rangle=\left(2 N_{m}-1\right)\left|N_{m}\right\rangle$,

$\sigma_{m}^{+}\left|N_{m}\right\rangle=\left(1-N_{m}\right)\left|(1-N)_{m}\right\rangle$,

$\sigma_{m}^{-}\left|N_{m}\right\rangle=N_{m}\left|(1-N)_{m}\right\rangle$.

\section{A.3. Kinetic equations in the Born approximation}

In deriving kinetic equations for the wire-internal populations $P_{m}(t),(m=1,2, \ldots, N)$ one has to notice the restriction on the limit of nonadiabatic transfer. Therefore, we can restrict ourself to the (second) Born approximation with respect to $V$. We replace $\mathscr{U}(\tau)$ by $\mathscr{U}^{(0)}(\tau)$ in the superoperator Eq. (A.21), and in line with relation Eq. (33) we have to derive an equation for $P\left(N_{m}, t\right)$. It follows from Eq. (A.28) that

$$
\begin{aligned}
\dot{P}\left(N_{m}, t\right)= & \frac{1}{\hbar^{2}} \sum_{s}\left|V_{m s}\right|^{2} \sum_{N_{s}}\left[N_{m}\left(1-N_{s}\right)+\left(1-N_{s}\right) N_{m}\right] \\
& \times\left\{P\left((1-N)_{m}, t\right) P\left((1-N)_{s}, t\right)\left(\mathscr{K}_{s m}+\mathscr{K}_{s m}^{*}\right)\right. \\
& \left.-P\left(N_{m}, t\right) P\left(N_{s}, t\right)\left(\mathscr{K}_{m s}+\mathscr{K}_{m s}^{*}\right)\right\} . \quad(\mathrm{A} .31)
\end{aligned}
$$

The correlation function reads as

$\mathscr{K}_{m s}=\int_{0}^{\infty} \mathrm{d} \tau \mathrm{e}^{-\mathrm{i}\left(\epsilon_{m}-\epsilon_{s}\right) \tau / \hbar} \operatorname{tr}_{\text {vib }}\left(W_{\mathrm{B}} \mathrm{e}^{\hat{R}_{m s}(\tau)} \mathrm{e}^{-\hat{R}_{m s}}\right)$,

where the $\epsilon_{m}$ are defined by Eq. (1), while

$\hat{R}_{m s}(\tau)=\exp \left(-\mathrm{i} H_{\mathrm{B}} \tau / \hbar\right) \hat{R}_{m s} \exp \left(\mathrm{i} H_{\mathrm{B}} \tau / \hbar\right)$.

The concrete form of $\hat{R}_{m s}$ is determined by the used type electron-vibrational coupling. In the case of a localcoupling model, we have $\hat{R}_{m s}=\hat{R}_{m}-\hat{R}_{s}$ with $\hat{R}_{m}$ defined by Eq. (16). When a Holstein model is employed then $\hat{R}_{m s}=\sum_{\lambda}\left(g_{m \lambda}-g_{s \lambda}\right)\left(b_{\lambda}^{+}-b_{\lambda}\right),\left(g_{m \lambda}=\kappa_{m \lambda} / \hbar \omega_{\lambda}\right)$.

According to the relations Eq. (33) it becomes possible to derive the following kinetic equation for the population $P_{m}(t)$ of the $m$ th site:

$$
\begin{aligned}
\dot{P}_{m}(t)= & -\frac{2 \pi}{\hbar} \sum_{s}\left|V_{m s}\right|^{2}\left\{P_{m}(t)\left(1-P_{s}(t)\right)(\mathrm{FC})_{m s}\right. \\
& \left.-\left(1-P_{m}(t)\right) P_{s}(t)(\mathrm{FC})_{s m}\right\} .
\end{aligned}
$$

Here, we introduced the Franck-Condon factor $(\mathrm{FC})_{m s}$ (as well as $(\mathrm{FC})_{s m}$ ) which has been obtained as a result of the averaging of the operators $H_{\mathrm{B}}$, Eq. (19) and $\hat{R}_{m s}$, Eq. (16) with respect to the vibrational states. It follows the standard expression

$$
(\mathrm{FC})_{m s}=\frac{1}{2 \pi \hbar} \int_{-\infty}^{+\infty} \mathrm{d} \tau \mathrm{e}^{-\mathrm{i}\left(\epsilon_{m}-\epsilon_{s}\right) \tau / \hbar} \mathrm{e}^{-Q_{m s}(\tau)},
$$

where the function

$$
\begin{aligned}
Q_{m s}(\tau)=2 & \int_{-\infty}^{+\infty} \frac{\mathrm{d} \omega}{\omega^{2}} J_{m s}(\omega)\left[\operatorname{coth} \frac{\hbar \omega}{k_{\mathrm{B}} T}(1-\cos \omega \tau)\right. \\
& -\mathrm{i} \sin \omega \tau],
\end{aligned}
$$

comprises the influence of the bath vibrational modes on the ET via the vibrational spectral density $J_{m s}(\omega)$. (This quantity is of basic importance for any calculation done on the spin-boson model $[3,5,6,61]$.) If the transition $m \rightarrow s$ is accompanied by the coupling to a single vibration with frequency $\omega_{\mathrm{f}}$ one can utilize the SongMarcus model [64], where we have $J_{m s}=$ $(1 / 2 \hbar) E_{\mathrm{f}}^{(\mathrm{r})} \omega \delta\left(\omega-\omega_{\mathrm{f}}\right)$ and where $E_{\mathrm{f}}^{(\mathrm{r})}$ denotes the reorganization energy for the $m \rightarrow s$ ET. As it well-known the Song-Marcus model leads to Jortner's form of the Franck-Condon factor [63]

$(\mathrm{FC})_{m s}=\frac{1}{\hbar \omega_{\mathrm{f}}} \Phi_{v_{\mathrm{f}}}$.

Here, we introduced

$$
\begin{aligned}
\Phi_{v_{\mathrm{f}}}= & \exp \left[-S_{\mathrm{f}} \operatorname{coth} \frac{\hbar \omega_{\mathrm{f}}}{k_{\mathrm{B}} T}\right]\left(\frac{1+n\left(\omega_{\mathrm{f}}\right)}{n\left(\omega_{\mathrm{f}}\right)}\right)^{v_{\mathrm{f}} / 2} \\
& \times I_{v_{\mathrm{f}}}\left(2 S_{\mathrm{f}}\left[n\left(\omega_{\mathrm{f}}\right)\left(1+n\left(\omega_{\mathrm{f}}\right)\right)\right]^{1 / 2}\right) .
\end{aligned}
$$

The expression contains the modified Bessel function $I_{v}(z)$, the Bose distribution function $n(\omega)=$ $\left[\exp \left(\hbar \omega / k_{\mathrm{B}} T\right)-1\right]^{-1}$, and we have set $S_{\mathrm{f}} \equiv E_{\mathrm{f}}^{(\mathrm{r})} / \hbar \omega_{\mathrm{f}}$. The energy $E_{\mathrm{f}}^{(\mathrm{r})}$ is the above mentioned reorganization energy of the $f$ th transition caused by the driving force $\Delta E_{\mathrm{f}}$, and $v_{\mathrm{f}} \equiv \Delta E_{\mathrm{f}} / \hbar \omega_{\mathrm{f}}$. For instance, at $m=1, s=0$ we put $E_{\mathrm{f}}^{(\mathrm{r})}=E_{1}^{(\mathrm{r})}, \Delta E_{\mathrm{f}}=\Delta E_{1}=\epsilon_{1}-\epsilon_{0}, \omega_{\mathrm{f}}=\omega_{1}, v_{\mathrm{f}}=v_{1}$.

The derived kinetic equations (A.34) depend nonlinearly on the site populations. However, it can be guaranteed for the reactions studied in the present paper that the wire-internal units are less populated during the ET process, i.e.

$P_{m}(t) \ll 1, \quad(m=1,2, \ldots, N)$,

is fulfilled at any time $t$. Resulting from this property a reduction to linear balance-like equations 
$\dot{P}_{m}(t)=-\sum_{s}\left[k_{m s} P_{m}(t)-k_{s m} P_{s}(t)\right]$

becomes possible. Here, each rate constant is defined as

$k_{m s}=\frac{2 \pi}{\hbar}\left|V_{m s}\right|^{2}(\mathrm{FC})_{m s}$,

with the Franck-Condon factor given by Eq. (A.35). A similar Born approximation results in a nonlinear kinetic equation valid for the population of the kth level of the left-electrode conduction band

$$
\begin{aligned}
\dot{P}_{\mathbf{L k}}(t)= & -\frac{2 \pi}{\hbar}\left|V_{\mathbf{L k}}\right|^{2}\left[P_{\mathbf{L} \mathbf{k}}(t)\left(1-P_{0}(t)\right)(\mathrm{FC})_{\mathbf{L k} 0}\right. \\
& \left.-\left(1-P_{\mathbf{L} \mathbf{k}}(t)\right) P_{0}(t)(\mathrm{FC})_{0 \mathbf{L k}}\right] .
\end{aligned}
$$

The Franck-Condon factors are defined via the above given general expression Eqs. (A.35) and (A.36), where now $m=\mathrm{L} \mathbf{k}, s=0$ or $m=0, s=\mathrm{Lk}$. Correspondingly, we obtain

$(\mathrm{FC})_{\mathrm{L} \mathbf{k} 0}=\frac{1}{2 \pi \hbar} \int_{\infty}^{+\infty} \mathrm{d} \tau \mathrm{e}^{-\mathrm{i}\left(E_{\mathbf{L k}}-\epsilon_{0}\right) \tau / \hbar} \mathrm{e}^{-Q_{0}(\tau)}$.

The function $Q_{0}(\tau)$ is defined by Eq. (A.36) through the spectral density $J_{0}(\omega)$. The only difference to $Q_{m s}(\tau)$ results from the fact that during the wire-internal ET $J_{m s}(\omega)$ is defined by the vibrations related to the two sites, $m$ and $s$, while for electrode-site or site-electrode transition the vibrations are relate to a single site, $m=0$ ( $m=N+1$ for a right electrode).

Let us sum up Eq. (A.42) with respect to $\mathbf{k}$ and bearing in mind that the total number of electrons occupying the conducting band of the left electrode is $N_{\mathrm{L}}(t)$, Eq. (27). For a bulk electrode, $N_{\mathrm{L}}(t)$ is given by a macroscopic large number. Practically, it does not change during the electron motion through the molecular wire. Therefore, we may set

$N_{\mathrm{L}}(t) \simeq N_{\mathrm{L}}=\sum_{\mathbf{k}} f_{\mathrm{Lk}}$,

where $f_{\mathrm{Lk}}=\left\{\exp \left[\left(E_{\mathrm{Lk}}-E_{\mathrm{F}}\right) / k_{\mathrm{B}} T\right]+1\right\}^{-1}$ is the equilibrium Fermi distribution function for the left electrode. According to Eq. (A.44) one can replace in the right part of Eq. (A.42) the population $P_{\mathrm{Lk}}(t)$ by the equilibrium distribution $f_{\mathrm{Lk}}$ once the summation of Eq. (A.42) over $\mathbf{k}$ has been carried out. This treatment leads us to the first equation of the set of equations (36) with the rate constants

$$
\begin{aligned}
& \chi_{\mathrm{L}}=\frac{2 \pi}{\hbar} \sum_{\mathbf{k}}\left|V_{\mathrm{Lk}}\right|^{2} f_{\mathrm{Lk}}(\mathrm{FC})_{\mathrm{L} \mathbf{k} 0}, \\
& \chi_{-\mathrm{L}}=\frac{2 \pi}{\hbar} \sum_{\mathbf{k}}\left|V_{\mathrm{Lk}}\right|^{2}\left(1-f_{\mathrm{Lk}}\right)(\mathrm{FC})_{0 \mathrm{~L} \mathbf{k}} .
\end{aligned}
$$

Analogously, one derives the last equation of set (36). The form of corresponding rate constants, $\chi_{R}$ and $\chi_{-R}$, follows from Eq. (A.45) replacing the symbol Lk by Rq.
A.4. Formation of a superexchange transfer rate between the terminal wire units

In the considered model of ET through a molecular wire the terminal wire units 0 and $N+1$ act as a donor and an acceptor with respect to internal wire units. Therefore, the terminal units do not only couple to the adjacent electrodes and the neighboring wire units but also one to another through the bridging sites $m=1,2, \ldots, N$. To derive the rate constants $k_{1} \equiv k_{0 N+1}$ and $k_{2} \equiv k_{N+10}$ which include the distant superexchange coupling between the sites 0 and $N+1$ one has to consider higher orders of perturbation theory with respect to the interaction $V$. Our aim is to derive a kinetic equation for the population $P_{0}(t)$ starting from the basic master Eq. (A.28). For the computation of the superoperator $\mathscr{R}$ responsible for the ET we utilize the expansion (A.25) and keep the necessary number of terms to couple site 0 to site $N+1$. The Born approximation results in kinetic processes which couple site 0 to the left electrode (with rate constants $\chi_{\mathrm{L}}$ and $\chi_{-\mathrm{L}}$ ) and to site 1 (with rate constants $\alpha_{0} \equiv k_{01}$ and $\beta_{0} \equiv k_{10}$ ). [Details on the formation of these processes are given in the previous subsection.]

Since the interaction $V$ is off-diagonal with respect to the electronic states, the first nonvanishing contribution following the second-order contribution stems from the time-propagation superoperator $\mathscr{U}^{(2)}(\tau)$. This contribution leads to two different contributions, a renormalization of the rate constants $\left(\chi_{\mathrm{L}}, \chi_{-\mathrm{L}}, \alpha_{0}\right.$ and $\left.\beta_{0}\right)$, and transitions between the sites 0 and 2 (with rates $k_{02}$ and $\left.k_{20}\right)$. Since nonadiabatic ET is considered the intersite matrix elements $V_{m m \pm 1}$ are small. Therefore, we can omit the rate constant renormalization. The rates expressions $k_{02}$ and $k_{20}$ are of the type of superexchange ET rates. But, if the number of internal wire units $N$ exceeds 1 , then site 2 belongs to the bridging units. Due to the large gap $\epsilon_{2}-\epsilon_{0}$ between bridging site 0 and 2 the rates $k_{02}$ and $k_{20}$ become too small to have any significant influence on the ET process (more details with respect to this question can be found in [51]). Of course, if the wire has only a single bridging unit one can not ignore the rates $k_{02}$ and $k_{20}$.

To determine the superexchange rates $k_{02}$ and $k_{20}$ at $N=1$ one has to expand Eq. (A.28) with $\mathscr{R}$ defined by $\mathscr{U}(\tau)=\mathscr{U}^{(2)}(\tau)$. There are a number of different terms which specify the superoperator $\mathscr{R}$, each of them contains the integration over $\tau, \tau_{1}$ and $\tau_{2}$. Let us consider a typical integral

$$
\begin{aligned}
A= & \frac{1}{\hbar^{4}} \int_{0}^{\infty} \mathrm{d} \tau \int_{0}^{\infty} \mathrm{d} \tau_{1} \int_{0}^{\infty} \mathrm{d} \tau_{2} \mathrm{e}^{-\mathrm{i}\left(\epsilon_{1}-\epsilon_{2}\right) \tau_{2} / \hbar} \mathrm{e}^{-\mathrm{i}\left(\epsilon_{0}-\epsilon_{2}\right) \tau_{1} / \hbar} \\
& \times \mathrm{e}^{-\mathrm{i}\left(\epsilon_{0}-\epsilon_{1}\right) \tau / \hbar} \operatorname{tr}_{\text {vib }}\left\{W_{\mathrm{B}} \tilde{V}_{01}\left(\tau+\tau_{1}\right) \tilde{V}_{21}(\tau) \tilde{V}_{10}(0)\right. \\
& \left.\times \tilde{V}_{12}\left(\tau+\tau_{1}+\tau_{2}\right)\right\},
\end{aligned}
$$


where we introduced $\quad \tilde{V}_{m m^{\prime}}(\tau)=V_{m m^{\prime}} \exp \left(-\mathrm{i} H_{\mathrm{B}} \tau / \hbar\right)$ $\exp \left(\hat{R}_{m m^{\prime}}\right) \exp \left(\mathrm{i} H_{\mathrm{B}} \tau / \hbar\right)$. It follows from the structure of the integrand that its oscillatory contributions are defined by the factors that contain the energy gaps $\Delta \epsilon_{12}=$ $\epsilon_{1}-\epsilon_{2}, \Delta \epsilon_{02}=\epsilon_{0}-\epsilon_{2}$ and $\Delta \epsilon_{10}=\epsilon_{1}-\epsilon_{0}$. The relation provide that at $N=1$ just the energy $\epsilon_{1}$ characterizes the position of the bridging LUMO-level. In line with the condition for deep tunneling one has to suppose the validity of the inequalities $\Delta \epsilon_{10} \gg\left|\Delta \epsilon_{02}\right|$ and $\Delta \epsilon_{12} \gg$ $\left|\Delta \epsilon_{02}\right|$. Following from this the characteristic oscillation time $\tau_{1} \sim \hbar / \Delta \epsilon_{02}$ strongly exceeds the characteristic times $\tau \sim \hbar / \Delta \epsilon_{10}$ and $\tau_{2} \sim \hbar / \Delta \epsilon_{12}$. This circumstance allows us to omit the times $\tau$ and $\tau_{2}$ in the interactions $\tilde{V}_{01}\left(\tau+\tau_{1}\right), \quad \tilde{V}_{21}(\tau)$ and $\tilde{V}_{12}\left(\tau+\tau_{1}+\tau_{2}\right)$. Noting that $\int_{0}^{\infty} \mathrm{d} \tau \exp (-\mathrm{i} x \tau)=-\mathrm{i} \lim _{\delta \rightarrow+0}(x-\mathrm{i} \delta)$ and thus $\int_{0}^{\infty} \mathrm{d} \tau \exp \left(\mathrm{i} \Delta \epsilon_{10} \tau\right) \int_{0}^{\infty} \mathrm{d} \tau_{2} \exp \left(-\mathrm{i} \Delta \epsilon_{12} \tau\right) \approx \hbar^{2} /\left(\Delta \epsilon_{10} \Delta \epsilon_{12}\right)$ the integral Eq. (A.46) is reduced to

$A \approx \frac{1}{\hbar^{2}} \frac{\left|V_{01} V_{12}\right|^{2}}{\Delta \epsilon_{10} \Delta \epsilon_{12}} \mathscr{K}_{02}$,

where the correlation function is defined by Eq. (A.32). The multiple time-integrals in the other terms contributing to the expansion have an analogous structure. Collecting all contributions it results a kinetic equation for $P_{0}(t)$ which is represented as a second equation in the set (36) with the superexchange rate constants

$k_{1(2)} \equiv k_{02(20)}=\frac{2 \pi}{\hbar} \frac{\left|V_{01} V_{12}\right|^{2}}{\Delta \epsilon_{10} \Delta \epsilon_{12}}(\mathrm{FC})_{02(20)}$.

At large numbers $N$ of internal wire units one has to take into consideration the superoperators $\mathscr{U}^{(4)}(\tau)$, $\mathscr{U}^{(6)}(\tau)$ and so on. The superoperator $\mathscr{U}^{(4)}(\tau)$ leads to a renormalization of the previously discussed rates $k_{01(10)}$ as well as $k_{02(20)}$, and generates the formation of the superexchange rate constants $k_{03}$ and $k_{30}$. But again, if site 3 belongs to a bridge we can neglect the kinetic processes characterized by the rate constants $k_{03}$ and $k_{30}$. Thus, one can omit the contribution of the superoperator $\mathscr{U}^{(4)}(\tau)$. Generally, one can neglect the contribution from any superoperator $\mathscr{U}^{(2(m-1))}(\tau)$, since it couples site 0 to any internal wire site $m=2,3, \ldots, N$. However, for $m=N+1$ we have to consider the respective contribution since we arrived at the superoperator $\mathscr{U}^{(2 N)}(\tau)$ which is responsible for the formation of the superexchange transfer process between the 0 th and the $N+1$ th terminal sites.

Thus, to derive the kinetic equations for $P\left(N_{0}, t\right)$ for the case of large energy gaps

$\Delta \epsilon_{m 0} \equiv \epsilon_{m}-\epsilon_{0}, \quad \Delta \epsilon_{m N+1} \equiv \epsilon_{m}-\epsilon_{N+1}$,

between the LUMO-levels of internal and terminal wire units, it is quite appropriate to only keep in the expansion (A.25) the superoperators $\mathscr{U}^{(0)}(\tau)$ and $\mathscr{U}^{(2 N)}(\tau)$. This results in the second equation of set (36) with rate expressions $k_{1(2)}=\frac{2 \pi}{\hbar}\left|V_{0 N+1}\right|^{2}(\mathrm{FC})_{0 N+1(N+10)}$.

Here, the square of the superexchange coupling reads

$\left|V_{0 N+1}\right|^{2}=\frac{\left|V_{1} V_{\mathrm{B}}^{N-1} V_{N}\right|^{2}}{\prod_{m=1}^{N} \Delta \epsilon_{m 0} \Delta \epsilon_{m N+1}}$,

while the Franck-Condon factors coincide with those given in Eq. (A.35) for $m=0, s=N+1$ or $m=N+1$, $s=0$. Note that Eq. (A.50) for the rate constants is valid if the condition

$\left|V_{m m \pm 1}\right| \ll \Delta \epsilon_{m 0}, \Delta \epsilon_{m N+1}$,

of deep tunneling is valid for all internal wire units $m=1,2, \ldots, N$. Take also into account that the superexchange coupling $\left|V_{0 N+1}\right|$ depends on the applied voltage $V$ through the gaps $\Delta \epsilon_{m 0}$ and $\Delta \epsilon_{m N+1}$. If, however, the voltage does not essentially alternate the gaps one can set $\Delta \epsilon_{10} \approx \Delta \epsilon_{20} \approx \cdots \Delta \epsilon_{N 0} \equiv \Delta E_{0}, \Delta \epsilon_{1 N+1} \approx \Delta \epsilon_{2 N+1} \approx$ $\Delta \epsilon_{N N+1} \equiv \Delta E_{N+1}$. This leads to the simplified form of the superexchange coupling so that we may set

$\left|V_{0 N+1}\right|^{2} \approx \frac{\left|V_{1} V_{N}\right|^{2}}{\Delta E_{0} \Delta E_{N+1}} \mathrm{e}^{-\zeta(N-1)}$,

where the diminishing constant $\zeta$ is defined by Eq. (64).

\section{References}

[1] R. Kubo, Y. Toyozawa, Prog. Theor. Phys. 13 (1955) 60.

[2] B. Fain, Theory of Rate Processes in Condensed Media, Springer, Berlin, 1980.

[3] A.O. Caldeira, A.J. Legget, Ann. Phys. (New York) 149 (1983) 374.

[4] A.J. Legget, S. Chakravarty, A. Dorsey, M.P.A. Fisher, A. Garg, W. Zwerger, Rev. Mod. Phys. 59 (1987) 1.

[5] M. Grifoni, P. Hänggi, Phys. Rep. 304 (1998) 229.

[6] U. Weiss, Quantum Dissipative Systems, second ed., World Scientific, Singapore, 1998.

[7] I.A. Goychuk, E.G. Petrov, V. May, Chem. Phys. Lett. 253 (1996) 428;

Phys. Rev. E 56 (1997) 1421.

[8] R.I. Cukier, C. Denk, M. Morillo, J. Chem. Phys. 111 (1999) 5408.

[9] M. Thorwart, M. Grifoni, P. Hänggi, Phys. Rev. Lett. 85 (2000) 860.

[10] M. Thorwart, M. Grifoni, P. Hänggi, Ann. Phys. (New York) 293 (2001) 15

[11] J. Jortner, M. Ratner (Eds.), Molecular Electronics, Blackwell Science, Oxford, 1997.

[12] A. Aviram, M. Ratner (Eds.), Molecular Electronics: Science and Technolgy, New York Academy of Sciencies, New York, 1998.

[13] V. Mujica, M. Kemp, M. Ratner, J. Chem. Phys. 101 (1994) 6849; V. Mujica, M. Kemp, M. Ratner, J. Chem. Phys. 101 (1994) 6856.

[14] E.G. Petrov, I.S. Tolokh, A.A. Demidenko, V.V. Gorbach, Chem. Phys. 193 (1995) 237.

[15] V. Mujica, M. Kemp, A. Roitberg, M. Ratner, J. Chem. Phys. 104 (1996) 7296. 
[16] M. Kemp, A. Roitberg, V. Mujica, T. Wanta, M.A. Ratner, J. Phys. Chem. 100 (1996) 8349.

[17] E.G. Petrov, I.S. Tolokh, V. May, Phys. Rev. Lett. 79 (1997) 4006.

[18] M. Magoga, C. Joachim, Phys. Rev. B 56 (1997) 4722.

[19] S.N. Yaliraki, M.A. Ratner, J. Chem. Phys. 109 (1998) 5036.

[20] P. Hänggi, M. Ratner, S. Yaliraki, (Eds.), Special issue: Processes in Molecular Wires; Chem. Phys. 281 (2002) 111.

[21] A. Nitzan, M.A. Ratner, Science 300 (2003) 1384.

[22] A. Aviram, M. Ratner, Chem. Phys. Lett. 29 (1974) 277.

[23] F.L. Carter (Ed.), Molecular Electronic Devices, Marcel Dekker, New York, 1982.

[24] A. Aviram (Ed.), Molecular Electronics: Science and Technology, AIP, New York, 1992.

[25] M.C. Petty, M.R. Bryce, D. Bloor (Eds.), An Introduction to Molecular Electronics, Oxford University Press, New York, 1995.

[26] R.M. Metzger, B. Chen, U. Höpfner, M.V. Lakshmikantham, D. Vuillaume, T. Kawai, X. Wu, H. Tashibana, T.V. Hughes, H. Sakurai, J.W. Baldwin, C. Hosch, M.P. Cava, L. Brehmer, G.J. Ashwell, J. Am. Chem. Soc. 119 (1997) 10455.

[27] J. Lehmann, S. Camalet, S. Kohler, P. Hänggi, Chem. Phys. Lett. 368 (2003) 282.

[28] A. Nitzan, Annu. Rev. Phys. Chem. 52 (2001) 681.

[29] M.A. Reed, C. Zhou, C.J. Miller, T.P. Burgin, J.M. Tour, Science 278 (1997) 252.

[30] W. Tian, S. Datta, S. Hong, R. Reifenberger, J.I. Henderson, C.I. Kubiak, J. Chem. Phys. 109 (1998) 2874.

[31] P.A. Derosa, J.M. Seminario, J. Phys. Chem. B 105 (2001) 471.

[32] H.B. Weber, J. Reichert, F. Weigend, R. Ochs, D. Beckmann, M. Mayor, R. Ahlrichs, H.V. Löhneysen, Chem. Phys. 281 (2002) 113.

[33] J. Chen, M.A. Reed, Chem. Phys. 281 (2002) 127.

[34] P. Damle, A.W. Ghosh, S. Datta, Chem. Phys. 281 (2002) 171.

[35] R. Landauer, Philos. Mag. 21 (1970) 863; Phys. Lett. A 85 (1981) 91.

[36] M. Büttiker, Phys. Rev. B 33 (1986) 3020.

[37] S. Datta, Electronic Transport in Mesoscopic Systems, Cambridge University Press, New York, 1995.

[38] H. Ness, A.J. Fisher, Phys. Rev. Lett. 83 (1999) 452.

[39] W.B. Dewis, M.R. Wasielewski, M. Ratner, W. Mujica, A. Nitzan, J. Phys. Chem. 101 (1997) 6158.

[40] D. Segal, A. Nitzan, W.B. Dewis, M.R. Wasielewski, M. Ratner, J. Phys. Chem. B 104 (2000) 3817.

[41] E.G. Petrov, P. Hänggi, Phys. Rev. Lett. 86 (2001) 2862.

[42] E.G. Petrov, V. May, P. Hänggi, Chem. Phys. 281 (2002) 211.
[43] J. Lehmann, G.-L. Ingold, P. Hänggi, Chem. Phys. 281 (2002) 199.

[44] D. Segal, A. Nitzan, Chem. Phys. 268 (2001) 315; Chem. Phys. 281 (2002) 235.

[45] A.K. Felts, W.T. Pollard, R.A. Friesner, J. Phys. Chem. 99 (1995) 2929.

[46] D. Segal, A. Nitzan, M. Ratner, W.B. Dewis, J. Chem. Phys. B $104(2000) 2790$.

[47] M. Bixon, J. Jortner, Chem. Phys. 281 (2002) 393.

[48] E.G. Petrov, I.S. Tolokh, V. May, J. Chem. Phys. 108 (1998) 4386.

[49] V. May, Phys. Rev. B 66 (2002) 245411.

[50] A unified description has been recently employed to derive an analytic form for an overall transfer rate characterizing a bridgemediated donor-acceptor ET [51,52].

[51] E.G. Petrov, Ye.V. Shevchenko, V.I. Teslenko, V. May, J. Chem. Phys. 115 (2001) 7107;

E.G. Petrov, V. May, J. Phys. Chem. A 105 (2001) 10176.

[52] E.G. Petrov, Ye.V. Shevchenko, V. May, Chem. Phys. 288 (2003) 269.

[53] A.S. Davydov, Quantum Mechanics, second ed., Pergamon Press, Oxford, 1976.

[54] T. Holstein, Ann. Phys. (New York) 8 (1959) 325; Ann. Phys. (New York) 8 (1959) 343.

[55] F.K. Fong, Theory of Molecular Relaxation, Wiley, New York, 1975.

[56] E.G. Petrov, Physics of Charge Transfer in Biosystems, Naukowa Dumka, Kiev, 1984 (in Russian).

[57] I.A. Goychuk, E.G. Petrov, V. May, J. Chem. Phys. 103 (1995) 4937.

[58] Interelectrode resonant tunneling becomes effective if molecular wire has molecular orbitals delocalized over all wire; just such a situation is happen when the current is mediated by a single molecule [29-34].

[59] K. Blum, Density Matrix Theory and Application, second ed., Plenum Press, New York, 1996.

[60] S. Mukamel, Principles of Nonlinear Optical Spectroscopy, Oxford University, New York, 1995.

[61] V. May, O. Kühn, Charge and Energy Transfer Dynamics in Molecular Systems, Wiley-VCH, Berlin, 1999.

[62] E.G. Petrov, V.V. Marchenko, Ya.R. Zelinskyy, Mol. Cryst. Liq. Cryst. 385 (2002) 121/1.

[63] J. Jortner, J. Chem. Phys. 64 (1976) 4860.

[64] X. Song, R.A. Marcus, J. Chem. Phys. 99 (1993) 7768; S. Tanaka, C.-P. Hsu, J. Chem. Phys. 111 (1999) 117. 